\title{
Extended collinearly-improved Balitsky-Kovchegov evolution equation in target rapidity
}

\author{
Wenchang Xiang, ${ }^{1,2}$ Yanbing Cai, ${ }^{1, *}$ Mengliang Wang, ${ }^{1}$ and Daicui Zhou ${ }^{3}$ \\ ${ }^{1}$ Guizhou Key Laboratory in Physics and Related Areas, and Guizhou Key Laboratory of Big Data Statistic \\ Analysis, Guizhou University of Finance and Economics, Guiyang 550025, China \\ ${ }^{2}$ Department of Physics, Guizhou University, Guiyang 550025, China \\ ${ }^{3}$ Key Laboratory of Quark and Lepton Physics (MOE), and Institute of Particle Physics, \\ Central China Normal University, Wuhan 430079, China
}

(Received 12 February 2021; accepted 21 June 2021; published 19 July 2021)

\begin{abstract}
An extended collinearly improved Balitsky-Kovchegov evolution equation in the target rapidity representation is derived by including the running coupling corrections during the expansion of the "real" $S$-matrix. We find that the running coupling brings important modifications to the evolution equation, as one can see that there are extra contributions to the evolution kernel once the running coupling is included. To identify the significance of the modifications, we numerically solve the evolution equation with and without the running coupling contributions during the $S$-matrix expansion. The numerical results show that the scattering amplitude is largely suppressed by the running coupling corrections, which indicate that one needs to consider the running coupling contributions during the derivation of the nonlinear evolution equation in the target rapidity representation.
\end{abstract}

DOI: 10.1103/PhysRevD.104.016018

\section{INTRODUCTION}

The color glass condensate (CGC) effective theory has proven to be a powerful theory to describe the strong interactions associated with high-energy and -density environments. The leading-order (LO) CGC calculations, which refer to the derivation of the nonlinear BalitskyJalilian-Marian-Iancu-McLerran-Weigert-LeonidovKovner (Balitsky-JIMWLK) equation [1-5] and its mean-field version known as the Balitsky-Kovchegov (BK) equation [1,6], have been able to qualitatively describe many phenomenological results, such as the reduced cross section in deep inelastic scattering (DIS) $[7,8]$ and single and double inclusive particle production in high-energy heavyion collisions [9-13]. However, it has been found that the LO CGC theory is insufficient for direct applications to the phenomenology, since the evolution speed of the scattering amplitude resulting from the $\mathrm{LO} \mathrm{BK}$ equation is too fast to quantitatively describe the experimental data $[14,15]$. To promote the LO CGC formalism to next-to-leading order (NLO) generally requires two key ingredients: the NLO BK

\footnotetext{
*myparticle@163.com
}

Published by the American Physical Society under the terms of the Creative Commons Attribution 4.0 International license. Further distribution of this work must maintain attribution to the author(s) and the published article's title, journal citation, and DOI. Funded by SCOAP. evolution equation and process-dependent NLO impact factors [16].

Concerning the NLO BK evolution equation, there have been tremendous developments in the calculations of the NLO corrections to the JIMWLK and BK equations in the literature over the past 15 years [17-23]. The pioneering work regarding the NLO corrections to the BK equation was performed by including the running coupling effect in Refs. [17,18]. A running coupling Balitsky-Kovchegov (rcBK) equation was obtained. It was shown that the growth of the dipole-hadron scattering amplitude resulting from the rcBK equation is significantly slowed down as compared to the LO one. Furthermore, it was found that the rcBK equation gives a rather successful description of the HERA data [14]. However, the running coupling effect is not the only large higher-order correction to the LO BK or JIMWLK equations. Except for the running coupling, the authors of Ref. [19] derived the full NLO BK evolution equation by including the quark loops, gluon loops, and the tree gluon diagrams with quadratic and cubic nonlinearities, and they obtained other contributions which are enhanced by double transverse logarithms. Unfortunately, the numerical study of the full NLO BK equation found that the equation is unstable, since the scattering amplitude can decrease as rapidity increases and can even turn negative for small dipoles [24,25]. The instability was traced back to the radiative corrections enhanced by double transverse logarithms.

To cure the instability problem, one has to resum the radiative corrections enhanced by double transverse 
logarithms to all orders. Two methods related to this specific issue were proposed [26,27], which use different recipes to impose a kinematical constraint on the successive gluon emissions during the rapidity evolution: (i) the kinematical constraint introduced in Ref. [26] leads to an evolution equation that is similar to the LO BKequation, but is nonlocal in rapidity $Y$; (ii) the resummation of the leading double logarithms has been performed in the evolution kernel in Ref. [27], resulting in a collinearly improved BalitskyKovchegov (ciBK) equation with a modified kernel, which is still local in rapidity $Y$. It has been shown that these two methods are equivalent in the resummation of the leading double transverse logarithms. It is known that both of them lead to a stable evolution equation and give good fits to the small- $x$ HERA data [28-30]. However, the authors of Ref. [31] found that there are some inconsistencies with the original analysis in Refs. [26,27]. They found that the instability of the full NLO BK is caused by the wrong choice of the rapidity variable which plays the role of the evolution time. Moreover, it has been found that the growth of the saturation exponent with the running coupling constant in the asymptotic region of rapidity has a strong scheme dependence on the resummation method, which should not occur in practice.

It is important to point out that the rapidity generally used in all of the above-mentioned BK equations is that of the projectile rapidity $Y$. In terms of the previous experience with the NLO Balitsky-Fadin-Kuraev-Lipatov (BFKL) equation [32-34], where similar issues were met and eventually cured, the evolution variable should be the rapidity of the hadronic target $\eta$, rather than the rapidity of the projectile. Inspired by the experience of handling the instability problem in the NLO BFKL equation, a novel method was proposed to derive the collinearly improved BK equation in the $\eta$ representation ( $\operatorname{ciBK}-\eta)$ from the corresponding evolution equation in the $Y$ representation by the change of variable $\eta=Y-\rho$, where $\rho$ is defined as $\rho=\ln \left(Q^{2} / Q_{0}^{2}\right)$, with $Q^{2}$ and $Q_{0}^{2}$ being the hard scale of the projectile and soft scale of the target, respectively [31]. As a consequence, the evolution variable in the ciBK- $\eta$ equation is the physical rapidity $\eta=\ln (1 / x)$, not the rapidity of the dipole projectile, and the ciBK- $\eta$ equation is stable and shows little scheme dependence on the resummation prescriptions. It is worth noting that the ciBK- $\eta$ is not at full NLO accuracy due to some evolution kernels missing running coupling contributions, which shall be discussed in the following.

Parallel to the developments in the NLO BK evolution equation, another effort in NLO contributions was also performed by including quark-antiquark Fock state contributions at one loop and quark-antiquark-gluon Fock state contributions to improve the photon impact factor in coordinate space and mixed space (longitudinal momentum, but transverse coordinate) [35-39]. The authors of Ref. [16] used a numerical method to estimate the importance of the NLO impact factor in the DIS structure functions in the mixed space representation, and they found that the NLO corrections can be significant. Moreover, the full NLO impact factor combined with three different improved evolution equations [kinematical constraint BK [26], ciBK [28], and canonical BK in $\eta$ (caBK- $\eta$ ) [31]] were used to meet, for the first time, HERA data very recently [40]. Surprisingly, it was found that the three dipole amplitudes result in a very similar description of the HERA data. The reason rendering to this unexpected result can be ascribed that all the attempts to the NLO BK evolution equations have ignored the energy-dependent part of the NLO impact factor. In addition, a simplified convolution of the solutions of the improved evolution equations with the NLO impact factor was used in Ref. [40], which could also be a cause of this unexpected result. In fact, one finds that the NLO BK equation cannot be disentangled from the NLO impact factor in the NLO DIS cross section arising from a NLO quark-antiquark-gluon Fock state [39]. From the above discussion, although we know that the energy dependence of the NLO impact factor plays a significant role in the improvement of the NLO BK equation, we will defer studying this alternative to future work due to the considerably increased complication of the derivation of the evolution equation. In this paper, we shall focus on extending the ciBK- $\eta$ equation derived in Ref. [31] to the full NLO case.

It is known that the LO BK approximation was used in the replacement of the first derivative in the expansions of the "real" $S$-matrix ${ }^{1}$ when the collinearly improved BK equation in the $Y$ representation was transformed to the $\eta$ representation [31], which leads to a loss of precision at the level of NLO accuracy. For completeness, the main purpose of this paper is to extend the collinearly improved BK equation in the $\eta$ representation to the full next-to-leadingorder case by including the running coupling corrections during the expansions of the "real" $S$-matrix. For later comparison, we first recall the derivation of the ciBK- $\eta$ equation with the LO BK approximation in the replacement of the first derivative in the expansion of the "real" $S$-matrix. Second, we derive an extended ciBK- $\eta$ equation by emphasizing the running coupling corrections in the expansion of the "real" $S$-matrix. We obtain an extended "canonical" Balitsky-Kovchegov equation (exBK- $\eta$ ) whose evolution kernel is modified by the running coupling corrections as compared to the caBK- $\eta$ equation. The exBK- $\eta$ equation is analytically solved in the saturation region. Its analytic solution shows that the exponent of the $S$-matrix has a linear dependence on rapidity instead of the quadratic rapidity dependence in the caBK- $\eta$ case, which obeys a similar law as results in the $Y$ representation in that the evolution speed of the dipole amplitude is suppressed

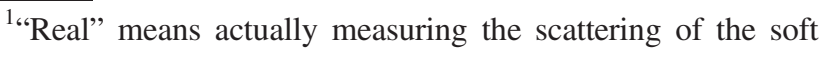
gluon.
} 
by the running coupling corrections. We compare our extended collinearly improved Balitsky-Kovchegov equation in $\eta$ with its original form to see how big the difference is. It is easy to find that there are eight extra terms resulting from the running coupling corrections, which indicate that the precision of the expansion of the $S$-matrix has a significant impact on the evolution equation.

Finally, we numerically solve the evolution equations in the $\eta$ representation to test the analytic outcomes mentioned above. The numerical results confirm our analytic findings. The saturation exponents $\bar{\lambda}$ are extracted from the solutions of the LO BK, caBK- $\eta$, and exBK- $\eta$ equations. As expected, the running coupling effect has a large influence on the evolution speed of the front, which largely suppresses the rapidity evolution of the dipole amplitude.

\section{LEADING ORDER AND RUNNING COUPLING BK EQUATIONS IN THE $Y$ REPRESENTATION}

In order to collect the basic elements of the dipole evolution equations, we give a brief review of the LO BK and rcBK equations in the $Y$ representation. These two equations shall be used in the Taylor expansion of the "real" $S$-matrix in the derivation of the collinearly improved BK equation in the $\eta$ representation in the next section.

\section{A. Leading-order BK equation}

We consider the high-energy scattering between a dipole which consists of a quark-antiquark pair moving in the positive direction of the longitudinal axis with momentum $\left(p^{+}, p^{-}, \boldsymbol{p}\right)$, and a hadronic target moving along the negative direction with momentum $\left(p_{0}^{+}, p_{0}^{-}, \boldsymbol{p}_{0}\right)$. The scattering is treated in the eikonal approximation, and thus the transverse coordinates of the quark $(\boldsymbol{x})$ and the antiquark $(y)$ are not modified by the collision. One can write the dipole scattering matrix as a correlator of two Wilson lines [19],

$$
S_{x y}(Y)=\frac{1}{N_{c}}\left\langle\operatorname{Tr}\left\{U(\boldsymbol{x}) U^{\dagger}(\boldsymbol{y})\right\}\right\rangle_{Y},
$$

where the $\langle\cdots\rangle_{Y}$ means the average over target gluon field configurations at $Y$. Here, $Y$ is the rapidity difference between the dipole and hadronic target,

$$
Y=\ln \frac{p^{+}}{p_{0}^{+}}=\ln \frac{2 p^{+} p_{0}^{-}}{Q_{0}^{2}}=\ln \frac{s}{Q_{0}^{2}},
$$

with the center-of-mass energy squared $s=2 p^{+} p_{0}^{-}$and the typical momentum of the target $Q_{0}$. The $U$ in Eq. (1) is the time-ordered Wilson line

$$
U(\boldsymbol{x})=\mathrm{P} \exp \left[i g \int \mathrm{d} x^{-} A^{+}\left(x^{-}, \boldsymbol{x}\right)\right],
$$

where $A^{+}\left(x^{-}, \boldsymbol{x}\right)$ is the gluon field of the hadronic target.

In the mean-field approximation, the rapidity evolution of the $S$-matrix satisfies the BK equation $[1,6]$

$$
\begin{aligned}
\frac{\partial}{\partial Y} S_{x y}(Y)= & \frac{\bar{\alpha}_{s}}{2 \pi} \int \mathrm{d}^{2} z \frac{(\boldsymbol{x}-\boldsymbol{y})^{2}}{(\boldsymbol{x}-\boldsymbol{z})^{2}(z-\boldsymbol{y})^{2}} \\
& \times\left[S_{x z}(Y) S_{z y}(Y)-S_{x y}(Y)\right],
\end{aligned}
$$

with $\bar{\alpha}_{s}=\alpha_{s} N_{c} / \pi$, and $\boldsymbol{x}, \boldsymbol{y}, \boldsymbol{z}$ are the transverse coordinates of the quark, antiquark, and emitted gluon, respectively. In the large- $N_{c}$ limit, Eq. (4) depicts the parent dipole $(\boldsymbol{x}, \boldsymbol{y})$ evolved into two daughter dipoles $(\boldsymbol{x}, \boldsymbol{z})$ and $(\boldsymbol{z}, \boldsymbol{y})$ as the rapidity increases. The first term on the right-hand side of Eq. (4) is called the "real" term which describes the two daughter dipoles scattering with the target simultaneously, and thus it is a nonlinear term. The second term on the right-hand side of Eq. (4) is referred to as the "virtual" term which depicts the survival probability of the original dipole at the time of scattering. Note that the BK equation resums only the leading-logarithmic $\alpha_{s} \ln (1 / x)$ corrections in the fixed-coupling case, and thus it is a $\mathrm{LO}$ evolution equation.

In the later sections of this paper, we shall be interested in the limiting form of the $S$-matrix in the saturation region where the dipole has a very large size, such that $r^{2} Q_{s}^{2} \gg 1$. Here, $Q_{s}$ is the typical transverse momentum of the saturated gluons and is rapidly increasing with $Y$. For later comparison, we analytically solve Eq. (4) in the following. In the saturation regime, the $S$-matrix approaches the blackdisk limit, $S \rightarrow 0$. Therefore, the quadratic term in $S$ in Eq. (4) can be neglected. Moreover, the saturation condition implies that the two daughter dipoles are larger than the typical transverse size $r_{s} \sim 1 / Q_{s}$. Therefore, Eq. (4) can reduce to

$$
\frac{\partial S(r, Y)}{\partial Y} \simeq-\frac{\bar{\alpha}_{s}}{2 \pi} \int_{1 / Q_{s}}^{r} \frac{\mathrm{d}^{2} r_{1} r^{2}}{r_{1}^{2} r_{2}^{2}} S(r, Y),
$$

where $r=|\boldsymbol{x}-\boldsymbol{y}|, r_{1}=|\boldsymbol{x}-\boldsymbol{z}|$, and $r_{2}=|\boldsymbol{z}-\boldsymbol{y}|$ are the transverse sizes of the parent dipole and two daughter dipoles, respectively. Further, one can find that the integral in Eq. (5) is governed by the case where one of the daughter dipoles is much smaller than the parent dipole, $r_{1} \ll r$ and $r_{2} \sim r$, or $r_{2} \ll r$ and $r_{1} \sim r$ [41,42]. We choose to work in the case where $r_{2} \sim r$, and thus Eq. (5) can be rewritten as

$$
\frac{\partial S(r, Y)}{\partial Y} \simeq-\bar{\alpha}_{s} \int_{1 / Q_{s}^{2}}^{r^{2}} \frac{\mathrm{d} r_{1}^{2}}{r_{1}^{2}} S(r, Y),
$$

where the right-hand side includes a factor of 2 to take into account that the smaller dipole with size $r_{1}$ can be any one of the two daughter dipoles. Now we carry out the integrals 
in Eq. (6), which yield the analytic solution of the LO BK equation as [41-44]

$$
S(r, Y)=\exp \left[-\frac{\lambda \bar{\alpha}_{s}^{2}}{2}\left(Y-Y_{0}\right)^{2}\right] S\left(r, Y_{0}\right),
$$

where we have used $\ln \left[r^{2} Q_{s}^{2}(Y)\right] \simeq \lambda \bar{\alpha}_{s}\left(Y-Y_{0}\right)$ with $Y_{0}$ being the rapidity scale at which $Q_{s}^{2}\left(Y_{0}\right)=1 / r^{2}$. Equation (7) is usually called the Levin-Tuchin formula, since it was first derived by them in Ref. [43]. From the above derivation, we know that the exponent in Eq. (7) is known only to leading double logarithmic accuracy, which means that the subleading terms are not under control. In addition, the exponent of the $S$-matrix has a quadratic dependence on rapidity, which renders the $S$-matrix too small at large rapidities; in the other words, the rapidity evolution speed of the dipole amplitude $N$ is too fast, with $N=1-S$. It has been recognized that the aforementioned drawbacks are the reasons why the LO BK equation is insufficient to describe the experimental data at HERA $[14,15,45]$. Therefore, one has to include the NLO corrections to the LO BK evolution equation, such as the running coupling effect which can suppress the evolution speed of the dipole amplitude by modifying the evolution kernel of the BK equation $[17,18]$.

\section{B. Running coupling BK equation}

It is known that the LO BK equation discussed above considers only the resummation of leading-logarithmic $\alpha_{s} \ln (1 / x)$ corrections with a fixed coupling. Beyond the leading-logarithmic approximation, there was significant progress in the BK evolution equation via the resummation of $\alpha_{s} N_{f}$ to all orders, which is called the running coupling corrections. The rcBK equation was derived independently by Balitsky in Ref. [17] and Kovchegov and Weigert in Ref. [18]. These two groups obtained analogous structures of the rcBK equation but with different evolution kernels. In this study, we shall use the Balitsky version of the kernel since it is favored by the HERA data [14]. Note that we do not plan to give details of the derivation of the rcBK equation, as they are beyond the scope of this paper. The rcBK equation reads

$\frac{\partial S(r, Y)}{\partial Y}=\int \mathrm{d}^{2} r_{1} K^{\mathrm{rc}}\left(r, r_{1}, r_{2}\right)\left[S\left(r_{1}, Y\right) S\left(r_{2}, Y\right)-S(r, Y)\right]$,

where $K^{\mathrm{rc}}\left(r, r_{1}, r_{2}\right)$ is the running coupling evolution kernel [17]

$$
\begin{aligned}
K^{\mathrm{rc}}\left(r, r_{1}, r_{2}\right)= & \frac{N_{c} \alpha_{s}\left(r_{\min }^{2}\right)}{2 \pi^{2}}\left[\frac{r^{2}}{r_{1}^{2} r_{2}^{2}}+\frac{1}{r_{1}^{2}}\left(\frac{\alpha_{s}\left(r_{1}^{2}\right)}{\alpha_{s}\left(r_{2}^{2}\right)}-1\right)\right. \\
& \left.+\frac{1}{r_{2}^{2}}\left(\frac{\alpha_{s}\left(r_{2}^{2}\right)}{\alpha_{s}\left(r_{1}^{2}\right)}-1\right)\right],
\end{aligned}
$$

with $r_{\min }=\min \left\{r, r_{1}, r_{2}\right\}$. There are several running coupling prescriptions in the literature [17,28,46-48]. At the very beginning, the argument of the running coupling $\alpha_{s}$ in the rcBK equation was interpreted as the transverse size of the parent dipole. Recently, it was found that the size of the smallest dipole is a proper argument of the coupling, and the smallest running coupling prescription is favored by the HERA data [28]. So, we shall use the smallest dipole running coupling prescription in this study. In addition, the running coupling at one-loop accuracy is used,

$$
\alpha_{s}\left(r^{2}\right)=\frac{1}{b \ln \left(\frac{1}{r^{2} \Lambda^{2}}\right)},
$$

with $b=\left(11 N_{c}-2 N_{f}\right) / 12 \pi$.

Now, let us analytically solve the rcBK equation. As it was done in the previous section, we solve the rcBK equation in the saturation region where the dipoles have a very large size, $r, r_{1}, r_{2} \geq 1 / Q_{s}$. The integral over $r_{1}$ in Eq. (8) is governed by the case where one of the daughter dipoles is much smaller than the parent one, while the rest of dipole has a similar size as the parent dipole, that is, $r_{1} \ll r$ and $r_{2} \sim r$, or $r_{2} \ll r$ and $r_{1} \sim r$. We choose to work in the first case, and thus Eq. (8) reduces to

$\frac{\partial S(r, Y)}{\partial Y}=\frac{1}{\pi} \int_{1 / Q_{s}}^{r} \frac{\mathrm{d}^{2} r_{1} \bar{\alpha}_{s}\left(r_{1}^{2}\right)}{r_{1}^{2}}\left[S\left(r_{1}, Y\right) S\left(r_{2}, Y\right)-S(r, Y)\right]$,

where the right-hand side includes a factor of 2 due to the fact that the smaller dipole can come from either of the two daughter dipoles. In the saturation region, the $S$-matrix approaches the black-disk limit, one has $S \rightarrow 0$, and thus the quadratic term in $S$ in Eq. (11) can be discarded. Equation (11) simplifies to

$$
\frac{\partial S(r, Y)}{\partial Y} \simeq-\int_{1 / Q_{s}^{2}}^{r^{2}} \frac{\mathrm{d} r_{1}^{2} \bar{\alpha}_{s}\left(r_{1}^{2}\right)}{r_{1}^{2}} S(r, Y) .
$$

Substituting Eq. (10) into Eq. (12), and performing the integrals over $r_{1}$ and $Y$, we can get the analytic solution of the rcBK equation as $[42,44]$

$$
\begin{aligned}
S(r, Y)= & \exp \left\{-\frac{N_{c}}{b \pi}\left(Y-Y_{0}\right)\left[\ln \left(\frac{\sqrt{\lambda^{\prime}\left(Y-Y_{0}\right)}}{\ln \frac{1}{r^{2} \Lambda^{2}}}\right)-\frac{1}{2}\right]\right\} \\
& \times S\left(r, Y_{0}\right),
\end{aligned}
$$

where the NLO saturation momentum is used,

$$
\ln \frac{Q_{s}^{2}}{\Lambda^{2}}=\sqrt{\lambda^{\prime}\left(Y-Y_{0}\right)}+\mathcal{O}\left(Y^{1 / 6}\right) .
$$

If one compares the solution of the rcBK equation (13) with the solution of the LO BK equation (7), it is easy to find that 
the quadratic rapidity dependence in the exponent of the $S$ matrix is replaced by a linear rapidity dependence due to the running coupling corrections. This outcome indicates that the evolution speed of the dipole amplitude is slowed down by the running coupling corrections. This finding is consistent with the theoretical expectations [17,49]. Furthermore, the phenomenological applications of the rcBK equation at HERA energies show that the rcBK equation gives a more reasonable description of the experimental data than the LO BK equation $[14,15]$.

\section{COLLINEARLY IMPROVED BK EQUATION IN THE $\boldsymbol{\eta}$ REPRESENTATION}

In the previous section, all of the dipole evolution equations were studied in the $Y$ representation. In this section, we shall discuss the dipole evolution equations in the $\eta$ representation for two key reasons. On the one hand, a recent study in Ref. [50] realized that the rapidity $\eta=$ $\ln (1 / x)$ of the hadronic target is the physical rapidity used in the DIS experiments at HERA, rather than the projectile rapidity $Y$. On the other hand, it was found that the instability of the full NLO BK equation is a consequence of the wrong choice of the "evolution time," which refers to the choice of the rapidity variable [31]. However, if one simply transforms the ciBK- $Y$ equation to the $\eta$ representation by the change of variable $\eta=Y-\rho$, the instability problem still exists due to NLO corrections enhanced by double collinear logarithms, but it is not as severe as the corresponding issue in $Y$. Based on the previous experience with the full NLO BK equation in $Y$, where a similar problem was identified and eventually cured by enforcing the time-ordering constraints on the successive gluon emissions, one can know that the ordering of the successive emissions in longitudinal momentum should be enforced in order to solve the instability problem in the $\eta$ representation. By doing this, the ciBK- $\eta$ equation was obtained in Ref. [31], which can be directly applied to phenomenology. However, it was found that the accuracy of the ciBK- $\eta$ equation needs to be improved, since the LO BK approximation was used in the replacement of the first derivative in the expansions of the "real" $S$-matrix when the ciBK- $\eta$ equation was derived, which leads to a loss of precision at the level of NLO accuracy. We would like to point out that in this study the BK evolution equations shall not be derived directly in the target evolved frame (in the $\eta$ representation), where the target is generally a nucleus whose wave function is saturated for modes softer than the saturation scale, which leads to extremely complicated calculations. In this section, we shall follow the same strategy as Ref. [31] by using a change of variable to derive the ciBK- $\eta$ equation at the level of running coupling when the "real" $S$-matrix is expanded. An extended collinearly improved BK equation in $\eta$ is obtained, which has the same structure as the ciBK- $\eta$ equation but with a runningcoupling-modified kernel. In the next section, we use the numerical method to solve the $\mathrm{caBK}-\eta$ and exBK- $\eta$ equations. The numerical results show that the running coupling corrections play a significant role in the suppression of the evolution of the dipole amplitude.

\section{A. Collinearly improved BK equation in $\eta$ : LO BK approximation in the expansion of the $S$-matrix}

To obtain the collinearly improved BK equation in $\eta$, we follow the same strategy used in Ref. [31] where the a change of variable is employed to transform the ciBK equation from the $Y$ representation to the $\eta$ representation.

We start with the full NLO BK equation in the $Y$ representation [17],

$$
\begin{aligned}
\frac{\partial S_{x y}(Y)}{\partial Y}= & \frac{\bar{\alpha}_{s}}{2 \pi} \int \mathrm{d}^{2} z \cdot\left(K_{0}+K_{q}+K_{g}\right) \cdot\left(S_{x z}(Y) S_{z y}(Y)-S_{x y}(Y)\right) \\
& +\frac{\bar{\alpha}_{s}^{2}}{8 \pi^{2}} \int \mathrm{d}^{2} \boldsymbol{u} \mathrm{d}^{2} z \cdot K_{1} \cdot\left(S_{x u}(Y) S_{u z}(Y) S_{z y}(Y)-S_{x u}(Y) S_{u y}(Y)\right) \\
& +\frac{\bar{\alpha}_{s}^{2}}{8 \pi^{2}} \frac{N_{f}}{N_{c}} \int \mathrm{d}^{2} u \mathrm{~d}^{2} z \cdot K_{f} \cdot\left(S_{x z}(Y) S_{u y}(Y)-S_{x u}(Y) S_{u y}(Y)\right),
\end{aligned}
$$

with

$$
\begin{gathered}
K_{0}=\frac{(x-y)^{2}}{(x-z)^{2}(z-y)^{2}}, \\
K_{q}=\frac{(x-y)^{2}}{(x-z)^{2}(z-y)^{2}} \bar{\alpha}_{s}\left[b \ln (x-y)^{2} \mu^{2}-b \frac{(x-z)^{2}-(y-z)^{2}}{(x-y)^{2}} \ln \frac{(x-z)^{2}}{(y-z)^{2}}\right] \\
K_{g}=\frac{(x-y)^{2}}{(x-z)^{2}(z-y)^{2}} \bar{\alpha}_{s}\left[\frac{67}{36}-\frac{\pi^{2}}{12}-\frac{5 N_{f}}{18 N_{c}}-\frac{1}{2} \ln \frac{(x-z)^{2}}{(x-y)^{2}} \ln \frac{(y-z)^{2}}{(x-y)^{2}}\right]
\end{gathered}
$$




$$
\begin{aligned}
K_{1}= & \frac{1}{(\boldsymbol{u}-\boldsymbol{z})^{4}}\left\{-2+\frac{(\boldsymbol{x}-\boldsymbol{u})^{2}(\boldsymbol{y}-\boldsymbol{z})^{2}+(\boldsymbol{x}-\boldsymbol{z})^{2}(\boldsymbol{y}-\boldsymbol{u})^{2}-4(\boldsymbol{x}-\boldsymbol{y})^{2}(\boldsymbol{u}-\boldsymbol{z})^{2}}{(\boldsymbol{x}-\boldsymbol{u})^{2}(\boldsymbol{y}-\boldsymbol{z})^{2}-(\boldsymbol{x}-\boldsymbol{z})^{2}(\boldsymbol{y}-\boldsymbol{u})^{2}} \ln \frac{(\boldsymbol{x}-\boldsymbol{u})^{2}(\boldsymbol{y}-\boldsymbol{z})^{2}}{(\boldsymbol{x}-\boldsymbol{z})^{2}(\boldsymbol{y}-\boldsymbol{u})^{2}}\right. \\
& \left.+\frac{(\boldsymbol{x}-\boldsymbol{y})^{2}(\boldsymbol{u}-\boldsymbol{z})^{2}}{(\boldsymbol{x}-\boldsymbol{u})^{2}(\boldsymbol{y}-\boldsymbol{z})^{2}}\left[1+\frac{(\boldsymbol{x}-\boldsymbol{y})^{2}(\boldsymbol{u}-\boldsymbol{z})^{2}}{(\boldsymbol{x}-\boldsymbol{u})^{2}(\boldsymbol{y}-\boldsymbol{z})^{2}-(\boldsymbol{x}-\boldsymbol{z})^{2}(\boldsymbol{y}-\boldsymbol{u})^{2}}\right] \ln \frac{(\boldsymbol{x}-\boldsymbol{u})^{2}(\boldsymbol{y}-\boldsymbol{z})^{2}}{(\boldsymbol{x}-\boldsymbol{z})^{2}(\boldsymbol{y}-\boldsymbol{u})^{2}}\right\},
\end{aligned}
$$

and

$$
K_{f}=\frac{1}{(\boldsymbol{u}-\boldsymbol{z})^{4}}\left[2-\frac{(\boldsymbol{x}-\boldsymbol{u})^{2}(\boldsymbol{y}-\boldsymbol{z})^{2}+(\boldsymbol{x}-\boldsymbol{z})^{2}(\boldsymbol{y}-\boldsymbol{u})^{2}-(\boldsymbol{x}-\boldsymbol{y})^{2}(\boldsymbol{u}-\boldsymbol{z})^{2}}{(\boldsymbol{x}-\boldsymbol{u})^{2}(\boldsymbol{y}-\boldsymbol{z})^{2}-(\boldsymbol{x}-\boldsymbol{z})^{2}(\boldsymbol{y}-\boldsymbol{u})^{2}} \ln \frac{(\boldsymbol{x}-\boldsymbol{u})^{2}(\boldsymbol{y}-\boldsymbol{z})^{2}}{(\boldsymbol{x}-\boldsymbol{z})^{2}(\boldsymbol{y}-\boldsymbol{u})^{2}}\right] .
$$

From Eq. (15), one can see that the full NLO BK equation has two main changes in the structure as compared to the LO BK equation. The first term on the right-hand side receives corrections from quark loops $\left(K_{q}\right)$ and the gluon loop $\left(K_{g}\right)$. The last two terms on the right-hand side refer to partonic fluctuations involving two additional partons except for the original parent partons (quark and antiquark). In the large- $N_{c}$ limit, they correspond to two consecutive emissions: the original parent dipole $(\boldsymbol{x}, \boldsymbol{y})$ emits a gluon at transverse coordinate $\boldsymbol{u}$, which is equivalent to two daughter dipoles $(\boldsymbol{x}, \boldsymbol{u})$ and $(\boldsymbol{u}, \boldsymbol{y})$; then, the dipole $(\boldsymbol{u}, \boldsymbol{y})$ emits a gluon at transverse coordinate $z$, which yields the dipoles $(\boldsymbol{u}, \boldsymbol{z})$ and $(\boldsymbol{z}, \boldsymbol{y})$.

\section{The "canonical" BK equation}

In order to transform Eq. (15) from the $Y$ representation into the $\eta$ representation, we need to change variables in terms of

$$
Y=\eta+\rho
$$

Now, we can rewrite the $S$-matrices in the $\eta$ representation as

$$
\begin{gathered}
S_{x y}(Y)=S_{x y}(\eta+\rho) \equiv \bar{S}_{x y}(\eta), \\
S_{x z}(Y)=S_{x z}(\eta+\rho)=S_{x z}\left(\eta+\ln \frac{(\boldsymbol{x}-\boldsymbol{z})^{2}}{(\boldsymbol{x}-\boldsymbol{y})^{2}}+\rho_{x z}\right) \\
=\bar{S}_{x z}\left(\eta+\ln \frac{(\boldsymbol{x}-\boldsymbol{z})^{2}}{(\boldsymbol{x}-\boldsymbol{y})^{2}}\right), \\
S_{z y}(Y)=S_{z y}(\eta+\rho)=S_{z y}\left(\eta+\ln \frac{(\boldsymbol{y}-z)^{2}}{(\boldsymbol{x}-\boldsymbol{y})^{2}}+\rho_{z y}\right) \\
=\bar{S}_{z y}\left(\eta+\ln \frac{(\boldsymbol{y}-\boldsymbol{z})^{2}}{(\boldsymbol{x}-\boldsymbol{y})^{2}}\right),
\end{gathered}
$$

where we have used

$$
\begin{aligned}
\rho & =\ln \left(\frac{Q^{2}}{Q_{0}^{2}}\right)=\ln \left(\frac{1}{(\boldsymbol{x}-\boldsymbol{y})^{2} Q_{0}^{2}}\right) \\
& =\ln \left(\frac{(\boldsymbol{x}-\boldsymbol{z})^{2}}{(\boldsymbol{x}-\boldsymbol{z})^{2}(\boldsymbol{x}-\boldsymbol{y})^{2} Q_{0}^{2}}\right)=\ln \left(\frac{(\boldsymbol{x}-\boldsymbol{z})^{2}}{(\boldsymbol{x}-\boldsymbol{y})^{2}}\right)+\rho_{x z},
\end{aligned}
$$

and

$$
\begin{aligned}
\rho & =\ln \left(\frac{Q^{2}}{Q_{0}^{2}}\right)=\ln \left(\frac{1}{(\boldsymbol{x}-\boldsymbol{y})^{2} Q_{0}^{2}}\right)=\ln \left(\frac{(z-y)^{2}}{(z-y)^{2}(\boldsymbol{x}-\boldsymbol{y})^{2} Q_{0}^{2}}\right) \\
& =\ln \left(\frac{(\boldsymbol{z}-\boldsymbol{y})^{2}}{(\boldsymbol{x}-\boldsymbol{y})^{2}}\right)+\rho_{z \boldsymbol{y}} .
\end{aligned}
$$

When one works at NLO in $\bar{\alpha}_{s}$, one can expand out the "real" $S$-matrices $\left(S_{x z}\right.$ and $\left.S_{z y}\right)$ in Taylor series, since the rapidity shift in the argument of $S$-matrices is typically much smaller than $\eta$ itself. The expansions of $S_{x z}$ and $S_{z y}$ can be expressed as

$$
\begin{aligned}
S_{x z}(Y) & =\bar{S}_{x z}\left(\eta+\ln \frac{(\boldsymbol{x}-\boldsymbol{z})^{2}}{(\boldsymbol{x}-\boldsymbol{y})^{2}}\right) \\
& \simeq \bar{S}_{x z}(\eta)+\ln \frac{(\boldsymbol{x}-\boldsymbol{z})^{2}}{(\boldsymbol{x}-\boldsymbol{y})^{2}} \frac{\partial \bar{S}_{x z}(\eta)}{\partial \eta}
\end{aligned}
$$

and

$$
\begin{aligned}
S_{z y}(Y) & =\bar{S}_{z y}\left(\eta+\ln \frac{(\boldsymbol{y}-\boldsymbol{z})^{2}}{(\boldsymbol{x}-\boldsymbol{y})^{2}}\right) \\
& \simeq \bar{S}_{z y}(\eta)+\ln \frac{(\boldsymbol{y}-\boldsymbol{z})^{2}}{(\boldsymbol{x}-\boldsymbol{y})^{2}} \frac{\partial \bar{S}_{z y}(\eta)}{\partial \eta}
\end{aligned}
$$

To the order of interest, we only need to keep the first nontrivial term in the above expansions, since each $\partial S / \partial \eta$ is formally suppressed by a power of $\bar{\alpha}_{s}$. For the derivative terms in Eqs. (27) and (28), in this subsection we use the LO BK equation to evaluate them, as was done in Ref. [31]: 


$$
\begin{aligned}
S_{x z}(Y) & =\bar{S}_{x z}\left(\eta+\ln \frac{(\boldsymbol{x}-\boldsymbol{z})^{2}}{(\boldsymbol{x}-\boldsymbol{y})^{2}}\right) \\
& \simeq \bar{S}_{x z}(\eta)+\frac{\bar{\alpha}_{s}}{2 \pi} \int \frac{\mathrm{d}^{2} \boldsymbol{u}(\boldsymbol{x}-\boldsymbol{z})^{2}}{(\boldsymbol{x}-\boldsymbol{u})^{2}(\boldsymbol{u}-\boldsymbol{z})^{2}} \ln \frac{(\boldsymbol{x}-\boldsymbol{z})^{2}}{(\boldsymbol{x}-\boldsymbol{y})^{2}}\left[\bar{S}_{x \boldsymbol{u}}(\eta) \bar{S}_{\boldsymbol{u z}}(\eta)-\bar{S}_{x z}(\eta)\right]
\end{aligned}
$$

and

$$
\begin{aligned}
S_{z y}(Y) & =\bar{S}_{z y}\left(\eta+\ln \frac{(\boldsymbol{y}-\boldsymbol{z})^{2}}{(\boldsymbol{x}-\boldsymbol{y})^{2}}\right) \\
& \simeq \bar{S}_{z \boldsymbol{y}}(\eta)+\frac{\bar{\alpha}_{s}}{2 \pi} \int \frac{\mathrm{d}^{2} \boldsymbol{u}(\boldsymbol{y}-\boldsymbol{z})^{2}}{(\boldsymbol{y}-\boldsymbol{u})^{2}(\boldsymbol{u}-\boldsymbol{z})^{2}} \ln \frac{(\boldsymbol{y}-\boldsymbol{z})^{2}}{(\boldsymbol{x}-\boldsymbol{y})^{2}}\left[\bar{S}_{z u}(\eta) \bar{S}_{\boldsymbol{u} y}(\eta)-\bar{S}_{z y}(\eta)\right]
\end{aligned}
$$

From the above equations, one can see that the rapidity shift in the argument of $S$-matrices is equivalent to adding a term of order $\mathcal{O}\left(\bar{\alpha}_{s}\right)$. We would like to point out that the LO BK equation is a rough approximation to estimate the derivative terms in Eqs. (27) and (28). Actually, the LO BK equation is insufficient due to its lower precision. To reach the relevant order of accuracy, one should use at least the level of the rcBK equation to evaluate the derivative terms, which shall be studied in the next subsection.

Now, substituting Eqs. (29) and (30) into Eq. (15), one can get a semifinished collinearly improved BK equation ${ }^{2}$ in $\eta$ [31],

$$
\begin{aligned}
\frac{\partial S_{x y}(\eta)}{\partial \eta}= & \frac{\bar{\alpha}_{s}}{2 \pi} \int \mathrm{d}^{2} \cdot K_{0} \cdot\left(\bar{S}_{x z}(\eta) \bar{S}_{z y}(\eta)-\bar{S}_{x y}(\eta)\right)+\frac{\bar{\alpha}_{s}}{2 \pi} \int \mathrm{d}^{2} z \cdot\left(K_{q}+K_{g}\right) \cdot\left(\bar{S}_{x z}(\eta) \bar{S}_{z y}(\eta)-\bar{S}_{x y}(\eta)\right) \\
& +\frac{\bar{\alpha}_{s}^{2}}{2 \pi^{2}} \int \mathrm{d}^{2} z \mathrm{~d}^{2} \boldsymbol{u} \cdot K_{l} \cdot \bar{S}_{x u}(\eta)\left(\bar{S}_{u z}(\eta) \bar{S}_{z y}(\eta)-\bar{S}_{u y}(\eta)\right) \\
& +\frac{\bar{\alpha}_{s}^{2}}{8 \pi^{2}} \int \mathrm{d}^{2} u \mathrm{~d}^{2} z \cdot K_{1} \cdot\left(\bar{S}_{x u}(\eta) \bar{S}_{u z}(\eta) \bar{S}_{z y}(\eta)-\bar{S}_{x u}(\eta) \bar{S}_{u y}(\eta)\right) \\
& +\frac{\bar{\alpha}_{s}^{2}}{8 \pi^{2}} \frac{N_{f}}{N_{c}} \int \mathrm{d}^{2} u \mathrm{~d}^{2} z \cdot K_{f} \cdot\left(\bar{S}_{x z}(\eta) \bar{S}_{u y}(\eta)-\bar{S}_{x u}(\eta) \bar{S}_{u y}(\eta)\right)
\end{aligned}
$$

with

$$
K_{l}=\frac{(x-y)^{2}}{(x-u)^{2}(u-z)^{2}(z-y)^{2}} \ln \frac{(u-y)^{2}}{(x-y)^{2}}
$$

The third term on the right-hand side of Eq. (31) results from the expansion of the "real" $S$-matrices in terms of rapidity shift with the $\mathrm{LO} \mathrm{BK}$ approximation in the evaluation of the derivative term. Moreover, we would like to point out that the third term comes purely from the change of variable and does not bring any new higher-order corrections to the evolution kernel. Note that the rapidity shift is neglected in the all NLO terms when Eq. (31) is derived. All of the terms of order $\mathcal{O}\left(\bar{\alpha}_{s}^{2}\right)$ in Eq. (15) are simply replaced like $S_{x z}(Y) \rightarrow \bar{S}_{x z}(\eta)$, since the rapidity shift takes a contribution of order $\mathcal{O}\left(\bar{\alpha}_{s}\right)$ which renders all of the NLO terms of order $\mathcal{O}\left(\bar{\alpha}_{s}^{3}\right)$. As we are only interested in

\footnotetext{
${ }^{2}$ The reason why we call it a semifinished equation is that it is still an unstable equation, which needs to do the resummations of the double collinear logarithms to totally get rid of the instability.
}

the terms up to the order of $\mathcal{O}\left(\bar{\alpha}_{s}^{2}\right)$, all of the terms beyond $\bar{\alpha}_{s}^{2}$ are abandoned in this paper. Moreover, two mathematical tricks are used when in deriving Eq. (31): (i) the property that the LO term is invariant under $\boldsymbol{x}-\boldsymbol{z} \rightarrow \boldsymbol{z}-\boldsymbol{y}$ is exploited to combine some terms, and (ii) the integral variables in the third term on the right-hand side of Eq. (31) are relabeled in terms of $\boldsymbol{u} \leftrightarrow \boldsymbol{z}$ in order to keep consistency with the physics picture mentioned above.

Equation (31) is a NLO evolution equation in the $\eta$ representation, which is a local equation in rapidity. By comparing Eq. (31) with Eq. (15), one can see that the difference between them is only an extra term (resulting from the change of variable) in the third line on the righthand side of Eq. (31). Originally, we would anticipate that the change of variable from $Y$ to $\eta$ can eliminate the instabilities occurring in Eq. (15). As expected, the instabilities caused by the violations of time-ordering (double anticollinear logarithms) disappear from Eq. (31), since the time-ordering property is automatically guaranteed in the $\eta$ evolution. Unfortunately, it has been shown that the change of variable triggers another type of instabilities associated with double collinear logarithms [31]. 
To cure the second instabilities mentioned above, it is known that the successive gluon emissions during the rapidity evolution have to be simultaneously ordered in lifetime and longitudinal momentum,

$$
\tau_{p} \gg \tau_{k} \gg \tau_{0},
$$

and

$$
\begin{aligned}
& p^{+} \gg k^{+} \gg p_{0}^{+} \Rightarrow \frac{2 p^{+}}{Q^{2}} Q^{2} \gg \frac{2 k^{+}}{\boldsymbol{k}^{2}} \boldsymbol{k}^{2} \gg \frac{2 p_{0}^{+}}{Q_{0}^{2}} Q_{0}^{2} \\
& \Rightarrow \tau_{p} Q^{2} \gg \tau_{\boldsymbol{k}} \boldsymbol{k}^{2} \gg \tau_{0} Q_{0}^{2},
\end{aligned}
$$

where $\left(p^{+}, p^{-}, \boldsymbol{p}\right),\left(p_{0}^{+}, p_{0}^{-}, \boldsymbol{p}_{0}\right)$, and $\left(k^{+}, k^{-}, \boldsymbol{k}\right)$ denote the light cone momenta of the projectile, target, and emitted gluon, respectively. The first constraint is automatically satisfied, as mentioned above. However, the second constraint may be violated when the radiated gluon is either too soft $\left(\boldsymbol{k}^{2} \ll Q_{0}^{2}\right)$ or too hard $\left(\boldsymbol{k}^{2} \gg Q^{2}\right)$. So, one has to put the constraint on the evolution equation. Before doing that, we need to rewrite the constraint (34) in a proper form. As we know,

$$
\rho=\ln \left(\frac{Q^{2}}{Q_{0}^{2}}\right), \quad Y=\ln \left(\frac{p^{+}}{p_{0}^{+}}\right),
$$

and

$$
\rho_{1}=\ln \left(\frac{\boldsymbol{k}^{2}}{Q_{0}^{2}}\right), \quad Y_{1}=\ln \left(\frac{k^{+}}{p_{0}^{+}}\right)
$$

Thus, the target rapidities can be expressed as

$$
\eta=Y-\rho=\ln \left(\frac{p^{+}}{p_{0}^{+}}\right)-\ln \left(\frac{Q^{2}}{Q_{0}^{2}}\right)=\ln \left(\frac{\tau_{p}}{\tau_{0}}\right)
$$

and

$$
\eta_{1}=Y_{1}-\rho_{1}=\ln \left(\frac{k^{+}}{p_{0}^{+}}\right)-\ln \left(\frac{\boldsymbol{k}^{2}}{Q_{0}^{2}}\right)=\ln \left(\frac{\tau_{k}}{\tau_{0}}\right) .
$$

Using Eqs. (37) and (38), one can rewrite the constraint (34) as

$$
\eta-\ln \left(\frac{\boldsymbol{k}^{2}}{Q^{2}}\right) \gg \eta_{1} \gg \ln \left(\frac{Q_{0}^{2}}{\boldsymbol{k}^{2}}\right) .
$$

Moreover, according to the lifetime constraint in Eq. (33), one can get

$$
\eta \gg \eta_{1} \gg 0 \text {. }
$$

Combining the two constraints (39) and (40), we obtain the final rapidity constraint as

$$
\min \left\{\eta, \eta-\ln \frac{\boldsymbol{k}^{2}}{Q^{2}}\right\}>\eta_{1}>\max \left\{0, \ln \frac{Q_{0}^{2}}{\boldsymbol{k}^{2}}\right\}
$$

By using Eqs. (35) and (36), the above equation can be written in another form as

$$
\Theta\left(-\rho_{1}\right)\left|\rho_{1}\right| \ll \eta_{1} \ll \eta-\Theta\left(\rho_{1}-\rho\right)\left(\rho_{1}-\rho\right),
$$

which is a proper form, and can be directly used.

Now, we apply the constraint (42) to the integral form of the BK equation and get

$$
\begin{aligned}
\bar{S}_{x y}(\eta)= & S_{x y}^{(0)}+\frac{\bar{\alpha}_{s}}{2 \pi} \int \frac{\mathrm{d}^{2} z(\boldsymbol{x}-\boldsymbol{y})^{2}}{(\boldsymbol{x}-\boldsymbol{z})^{2}(\boldsymbol{z}-\boldsymbol{y})^{2}} \\
& \times \int_{\Theta\left(-\rho_{1}\right)\left|\rho_{1}\right|}^{\eta-\Theta\left(\rho_{1}-\rho\right)\left(\rho_{1}-\rho\right)} \mathrm{d} \eta_{1}\left[\bar{S}_{x z}\left(\eta_{1}\right) \bar{S}_{z y}\left(\eta_{1}\right)-\bar{S}_{x y}\left(\eta_{1}\right)\right],
\end{aligned}
$$

which turns to differential format as

$$
\begin{aligned}
\frac{\partial \bar{S}_{x y}(\eta)}{\partial \eta}= & \frac{\bar{\alpha}_{s}}{2 \pi} \int \frac{\mathrm{d}^{2} z(\boldsymbol{x}-\boldsymbol{y})^{2}}{(\boldsymbol{x}-\boldsymbol{z})^{2}(z-\boldsymbol{y})^{2}} \\
& \times \Theta\left(\eta-\delta_{x y z}\right) \Theta\left(\eta-\Theta\left(-\rho_{1}\right)\left|\rho_{1}\right|\right) \\
& \times\left[\bar{S}_{x z}\left(\eta-\delta_{x y z}\right) \bar{S}_{z \boldsymbol{y}}\left(\eta-\delta_{x y z}\right)-\bar{S}_{x y}\left(\eta-\delta_{x y z}\right)\right],
\end{aligned}
$$

where the rapidity shift $\delta_{x y z}$ is

$$
\delta_{x y z}=\max \left\{0, \ln \frac{(\boldsymbol{x}-\boldsymbol{y})^{2}}{\min \left\{(\boldsymbol{x}-\boldsymbol{z})^{2},(\boldsymbol{z}-\boldsymbol{y})^{2}\right\}}\right\} .
$$

It has been checked that Eq. (44) has little scheme dependence on the prescription of the rapidity shift. In this study, we choose to work with the "canonical" one [31],

$$
\begin{array}{r}
\bar{S}_{x z}\left(\eta-\delta_{x y z}\right) \bar{S}_{z y}\left(\eta-\delta_{x y z}\right)-\bar{S}_{x y}\left(\eta-\delta_{x y z}\right) \\
\quad \rightarrow \bar{S}_{x z}\left(\eta-\delta_{x z ; r}\right) \bar{S}_{z y}\left(\eta-\delta_{z y ; r}\right)-\bar{S}_{x y}(\eta),
\end{array}
$$

with

$$
\delta_{x z ; r}=\max \left\{0, \ln \frac{r^{2}}{(x-z)^{2}}\right\}
$$

and

$$
\delta_{z y ; r}=\max \left\{0, \ln \frac{r^{2}}{(z-y)^{2}}\right\} .
$$

Substituting Eq. (46) into Eq. (44), one gets the caBK- $\eta$ equation as [31] 


$$
\begin{aligned}
\frac{\partial \bar{S}_{x y}(\eta)}{\partial \eta}= & \frac{\bar{\alpha}_{s}}{2 \pi} \int \mathrm{d}^{2} z \cdot K_{0} \cdot \Theta\left(\eta-\delta_{x y z}\right) \\
& \times\left[\bar{S}_{x z}\left(\eta-\delta_{x z ; r}\right) \bar{S}_{z y}\left(\eta-\delta_{z y ; r}\right)-\bar{S}_{x y}(\eta)\right],
\end{aligned}
$$

which is a nonlocal evolution equation in rapidity $\eta$.

\section{Full collinear improved BK equation at $\mathcal{O}\left(\bar{\alpha}_{s}^{2}\right)$}

The caBK- $\eta$ equation can be generalized to full NLO accuracy by adding all of the NLO corrections at $\mathcal{O}\left(\bar{\alpha}_{s}^{2}\right)$ in Eq. (31). Before adding those terms, we need to subtract the $\mathcal{O}\left(\bar{\alpha}_{s}^{2}\right)$ piece in Eq. (49). The $\mathcal{O}\left(\bar{\alpha}_{s}^{2}\right)$ piece in Eq. (49) can be identified by expanding the $\bar{S}_{x z}\left(\eta-\delta_{x z ; r}\right) \bar{S}_{z y}\left(\eta-\delta_{z y ; r}\right)$ term, as we have done in Eqs. (29) and (30). One finds that the $\mathcal{O}\left(\bar{\alpha}_{s}^{2}\right)$ piece is

$$
\begin{aligned}
& -\frac{\bar{\alpha}_{s}^{2}}{2 \pi^{2}} \int \frac{\mathrm{d}^{2} z \mathrm{~d}^{2} \boldsymbol{u}(\boldsymbol{x}-\boldsymbol{y})^{2}}{(\boldsymbol{x}-\boldsymbol{u})^{2}(\boldsymbol{u}-\boldsymbol{z})^{2}(z-\boldsymbol{y})^{2}} \\
& \quad \times \delta_{u y ; r} \bar{S}_{x \boldsymbol{u}}(\eta)\left[\bar{S}_{u z}(\eta) \bar{S}_{z y}(\eta)-\bar{S}_{u y}(\eta)\right] .
\end{aligned}
$$

Adding the $\mathcal{O}\left(\bar{\alpha}_{s}^{2}\right)$ pieces from Eq. (31) to Eq. (49) and subtracting the above $\mathcal{O}\left(\bar{\alpha}_{s}^{2}\right)$ piece, one obtains the ciBK- $\eta$ equation as [31]

$$
\begin{aligned}
\frac{\partial S_{x y}(\eta)}{\partial \eta}= & \frac{\bar{\alpha}_{s}}{2 \pi} \int \mathrm{d}^{2} z \cdot K_{0} \cdot \Theta\left(\eta-\delta_{x y z}\right)\left(\bar{S}_{x z}\left(\eta-\delta_{x z ; r}\right) \bar{S}_{z y}\left(\eta-\delta_{z y ; r}\right)-\bar{S}_{x y}(\eta)\right) \\
& +\frac{\bar{\alpha}_{s}}{2 \pi} \int \mathrm{d}^{2} z \cdot\left(K_{q}+K_{g}\right)\left(\bar{S}_{x z}(\eta) \bar{S}_{z y}(\eta)-\bar{S}_{x y}(\eta)\right) \\
& +\frac{\bar{\alpha}_{s}^{2}}{2 \pi^{2}} \int \mathrm{d}^{2} z \mathrm{~d}^{2} \boldsymbol{u} \cdot K_{2} \cdot \bar{S}_{x u}(\eta)\left(\bar{S}_{u z}(\eta) \bar{S}_{z y}(\eta)-\bar{S}_{u y}(\eta)\right) \\
& +\frac{\bar{\alpha}_{s}^{2}}{8 \pi^{2}} \int \mathrm{d}^{2} \boldsymbol{u} \mathrm{d}^{2} z \cdot K_{1} \cdot\left(\bar{S}_{x u}(\eta) \bar{S}_{u z}(\eta) \bar{S}_{z y}(\eta)-\bar{S}_{x u}(\eta) \bar{S}_{u y}(\eta)\right) \\
& +\frac{\bar{\alpha}_{s}^{2}}{8 \pi^{2}} \frac{N_{f}}{N_{c}} \int \mathrm{d}^{2} \boldsymbol{u} \mathrm{d}^{2} z \cdot K_{f} \cdot\left(\bar{S}_{x z}(\eta) \bar{S}_{u y}(\eta)-\bar{S}_{x u}(\eta) \bar{S}_{u y}(\eta)\right)
\end{aligned}
$$

with

$$
K_{2}=K_{l}+\frac{(\boldsymbol{x}-\boldsymbol{y})^{2}}{(\boldsymbol{x}-\boldsymbol{u})^{2}(\boldsymbol{u}-\boldsymbol{z})^{2}(\boldsymbol{z}-\boldsymbol{y})^{2}} \delta_{u \boldsymbol{u} ; r} .
$$

We would like to note that $K_{2}$ results from the change of variable and does not yield new higher-order corrections to the evolution kernel. One can see that Eq. (51) now does not include double collinear logarithms. These double logarithms are included in the first line on the right-hand side of Eq. (51) through the rapidity shift. In addition, the double anticollinear logarithm term in the kernel $K_{g}$ in the second line on the right-hand side of Eq. (51) is canceled by a relative piece generated by the integral over $\boldsymbol{u}$ in the third line. All of the unstable factors are under control in Eq. (51), and so it is a stable equation that can be directly applied to the phenomenological studies.

\section{Analytic solution to the caBK- $\eta$ equation in the saturation region}

Let us analytically solve the caBK- $\eta$ equation in the saturation region. In this regime, one of the daughters is much smaller than the other one, while the larger daughter dipole has a comparable size to the parent dipole. We know that the nonlocality is only important for the $S$-matrix that is associated with the smaller dipole, and thus Eq. (49) can be simplified to

$$
\begin{aligned}
\frac{\partial \bar{S}(r, \eta)}{\partial \eta} \simeq & 2 \frac{\bar{\alpha}_{s}}{2 \pi} \bar{S}(r, \eta) \int_{1 / Q_{s}}^{r} \frac{\mathrm{d}^{2} z}{z^{2}} \Theta\left(\eta-\ln \frac{r^{2}}{z^{2}}\right) \\
& \times\left[\bar{S}\left(z, \eta-\ln \frac{r^{2}}{z^{2}}\right)-1\right],
\end{aligned}
$$

where a factor of 2 is taken into account due to the fact that the smaller dipole can come from either of the two daughter dipoles, and $Q_{s}$ is the saturation momentum which is associated with $\bar{Q}_{s}$ as $r^{2} Q_{s}^{2}=\left(r^{2} \bar{Q}_{s}^{2}\right)^{1 /(1+\bar{\lambda})}[31]$. For simplicity, we denote the size of the smaller dipole in Eq. (53) as $z$.

To solve Eq. (53) in the saturation region, we follow two rules to do the calculations: (i) the saturation condition requires that the dipole size $z$ is larger than the typical size $1 / Q_{s}$ [lower integral bound in Eq. (53)], which leads to the "real" $S$-matrix $S\left(z, \eta-\ln \frac{r^{2}}{z^{2}}\right)$ being negligibly small; (ii) the integral over $z$ becomes logarithmic when $z$ is much smaller than $r$. Applying the two rules, Eq. (53) reduces to

$$
\frac{\partial \bar{S}(r, \eta)}{\partial \eta} \simeq-\bar{\alpha}_{s} \bar{S}(r, \eta) \int_{1 / Q_{s}^{2}}^{r^{2}} \frac{\mathrm{d} z^{2}}{z^{2}},
$$

whose solution is 


$$
\bar{S}(r, \eta)=\exp \left[-\frac{\bar{\alpha}_{s}^{2}}{2} \frac{\bar{\lambda}}{1+\bar{\alpha}_{s} \bar{\lambda}}\left(\eta-\eta_{0}\right)^{2}\right] \bar{S}\left(r, \eta_{0}\right)
$$

where we have assumed the saturation momentum in the $\eta$ representation as $\bar{Q}_{s}^{2}=Q_{0}^{2} \exp (\bar{\lambda} \eta)$. By comparing Eq. (55) with Eq. (7), one can see that they have similar forms, but the solution of the caBK- $\eta$ equation has an extra suppression factor in the exponent, which leads to the evolution speed of the dipole amplitude being slowed down. The numerical solutions of these two equations shall be obtained in the next section, where the numerical calculations support the aforementioned analytical result.

\section{B. Collinearly improved BK equation in $\eta$ : $\mathrm{rcBK}$ approximation in the expansion of the $S$-matrix}

In the previous subsection, the first derivative terms in the Taylor expansions in Eqs. (27) and (28) were approximately replaced by the LO BK equation, which is insufficient. To achieve the relevant order of accuracy, we shall derive the collinearly improved BK equation in $\eta$ by using the rcBK equation (8) to replace the LO BK equation (4) in the expression for the first derivative terms.

\section{Extended caBK- $\eta$ equation}

Using the rcBK equation, one can reexpand the $S$-matrices in Eqs. (27) and (28) as

$$
\begin{aligned}
S_{x z}(Y) & =\bar{S}_{x z}\left(\eta+\ln \frac{(\boldsymbol{x}-\boldsymbol{z})^{2}}{(\boldsymbol{x}-\boldsymbol{y})^{2}}\right) \\
& \simeq \bar{S}_{x z}(\eta)+\ln \frac{(\boldsymbol{x}-\boldsymbol{z})^{2}}{(\boldsymbol{x}-\boldsymbol{y})^{2}} \frac{\partial \bar{S}_{x z}(\eta)}{\partial \eta} \\
& \simeq \bar{S}_{x z}(\eta)+\int \mathrm{d}^{2} \boldsymbol{u} K^{\mathrm{rc}}(\boldsymbol{x}, \boldsymbol{z}, \boldsymbol{u}) \ln \frac{(\boldsymbol{x}-\boldsymbol{z})^{2}}{(\boldsymbol{x}-\boldsymbol{y})^{2}}\left[\bar{S}_{x u}(\eta) \bar{S}_{u z}(\eta)-\bar{S}_{x z}(\eta)\right]
\end{aligned}
$$

and

$$
\begin{aligned}
S_{z y}(Y) & =\bar{S}_{z y}\left(\eta+\ln \frac{(\boldsymbol{y}-\boldsymbol{z})^{2}}{(\boldsymbol{x}-\boldsymbol{y})^{2}}\right) \\
& \simeq \bar{S}_{z y}(\eta)+\ln \frac{(\boldsymbol{y}-\boldsymbol{z})^{2}}{(\boldsymbol{x}-\boldsymbol{y})^{2}} \frac{\partial \bar{S}_{x z}(\eta)}{\partial \eta} \\
& \simeq \bar{S}_{z y}(\eta)+\int \mathrm{d}^{2} \boldsymbol{u} K^{\mathrm{rc}}(z, \boldsymbol{y}, \boldsymbol{u}) \ln \frac{(\boldsymbol{z}-\boldsymbol{y})^{2}}{(\boldsymbol{x}-\boldsymbol{y})^{2}}\left[\bar{S}_{z u}(\eta) \bar{S}_{u y}(\eta)-\bar{S}_{z y}(\eta)\right]
\end{aligned}
$$

with the running coupling evolution kernel

$$
K^{\mathrm{rc}}(\boldsymbol{x}, \boldsymbol{y}, \boldsymbol{z})=\frac{\bar{\alpha}_{s}}{2 \pi}\left[\frac{(\boldsymbol{x}-\boldsymbol{y})^{2}}{(\boldsymbol{x}-\boldsymbol{z})^{2}(\boldsymbol{y}-\boldsymbol{z})^{2}}+\frac{1}{(\boldsymbol{x}-z)^{2}}\left(\frac{\alpha_{s}^{x z}}{\alpha_{s}^{y z}}-1\right)+\frac{1}{(\boldsymbol{y}-z)^{2}}\left(\frac{\alpha_{s}^{y z}}{\alpha_{s}^{x z}}-1\right)\right],
$$

where we use the shorthand notation $\alpha_{s}^{x z}=\alpha_{s}\left((x-z)^{2}\right)$ and similarly for others. Note that Eq. (58) is another form of Eq. (9), expressed in terms of transverse coordinates of the dipoles. Substituting Eqs. (56) and (57) into Eq. (15), we obtain a semifinished local collinearly improved BK equation in $\eta$ after some complicated algebraic calculations (for the detailed derivation, see Appendix A),

$$
\begin{aligned}
\frac{\partial S_{x y}(\eta)}{\partial \eta}= & \frac{\bar{\alpha}_{s}}{2 \pi} \int \mathrm{d}^{2} z \cdot K_{0} \cdot\left(\bar{S}_{x z}(\eta) \bar{S}_{z y}(\eta)-\bar{S}_{x y}(\eta)\right)+\frac{\bar{\alpha}_{s}}{2 \pi} \int \mathrm{d}^{2} z \cdot\left(K_{q}+K_{g}\right)\left(\bar{S}_{x z}(\eta) \bar{S}_{z y}(\eta)-\bar{S}_{x y}(\eta)\right) \\
& +\frac{\bar{\alpha}_{s}^{2}}{2 \pi^{2}} \int \mathrm{d}^{2} z \mathrm{~d}^{2} \boldsymbol{u} \cdot K_{\mathrm{rc}} \cdot \bar{S}_{x u}(\eta)\left(\bar{S}_{u z}(\eta) \bar{S}_{z y}(\eta)-\bar{S}_{u y}(\eta)\right)+\frac{\bar{\alpha}_{s}^{2}}{8 \pi^{2}} \int \mathrm{d}^{2} u \mathrm{~d}^{2} z \cdot K_{1} \cdot\left(\bar{S}_{x u}(\eta) \bar{S}_{u z}(\eta) \bar{S}_{z y}(\eta)-\bar{S}_{x u}(\eta) \bar{S}_{u y}(\eta)\right) \\
& +\frac{\bar{\alpha}_{s}^{2}}{8 \pi^{2}} \frac{N_{f}}{N_{c}} \int \mathrm{d}^{2} \boldsymbol{u} \mathrm{d}^{2} z \cdot K_{f} \cdot\left(\bar{S}_{x z}(\eta) \bar{S}_{u y}(\eta)-\bar{S}_{x u}(\eta) \bar{S}_{u y}(\eta)\right)
\end{aligned}
$$

with 


$$
\begin{aligned}
K_{\mathrm{rc}}= & \ln \frac{(\boldsymbol{u}-\boldsymbol{y})^{2}}{(\boldsymbol{x}-\boldsymbol{y})^{2}}\left[\frac{(\boldsymbol{x}-\boldsymbol{y})^{2}}{(\boldsymbol{x}-\boldsymbol{u})^{2}(\boldsymbol{u}-\boldsymbol{z})^{2}(\boldsymbol{y}-\boldsymbol{z})^{2}}+\frac{(\boldsymbol{x}-\boldsymbol{y})^{2}}{(\boldsymbol{x}-\boldsymbol{u})^{2}(\boldsymbol{y}-\boldsymbol{u})^{2}} \frac{1}{(\boldsymbol{u}-z)^{2}}\left(\frac{\alpha_{s}^{u z}}{\alpha_{s}^{y z}}-1\right)\right. \\
& +\frac{(\boldsymbol{x}-\boldsymbol{y})^{2}}{(\boldsymbol{x}-\boldsymbol{u})^{2}(\boldsymbol{y}-\boldsymbol{u})^{2}(\boldsymbol{y}-\boldsymbol{z})^{2}}\left(\frac{\alpha_{s}^{y z}}{\alpha_{s}^{u z}}-1\right)+\frac{(\boldsymbol{u}-\boldsymbol{y})^{2}}{(\boldsymbol{x}-\boldsymbol{u})^{2}(\boldsymbol{u}-\boldsymbol{z})^{2}(\boldsymbol{y}-\boldsymbol{z})^{2}}\left(\frac{\alpha_{s}^{x u}}{\alpha_{s}^{y u}}-1\right) \\
& +\frac{1}{(\boldsymbol{x}-\boldsymbol{u})^{2}(\boldsymbol{u}-\boldsymbol{z})^{2}}\left(\frac{\alpha_{s}^{x u}}{\alpha_{s}^{y u}}-1\right)\left(\frac{\alpha_{s}^{u z}}{\alpha_{s}^{y z}}-1\right)+\frac{1}{(\boldsymbol{x}-\boldsymbol{u})^{2}(\boldsymbol{y}-z)^{2}}\left(\frac{\alpha_{s}^{x u}}{\alpha_{s}^{y u}}-1\right)\left(\frac{\alpha_{s}^{y z}}{\alpha_{s}^{u z}}-1\right) \\
& +\frac{1}{(\boldsymbol{u}-\boldsymbol{z})^{2}(\boldsymbol{y}-\boldsymbol{z})^{2}}\left(\frac{\alpha_{s}^{y u}}{\alpha_{s}^{x u}}-1\right)+\frac{1}{(\boldsymbol{y}-\boldsymbol{u})^{2}(\boldsymbol{u}-\boldsymbol{z})^{2}}\left(\frac{\alpha_{s}^{y u}}{\alpha_{s}^{x u}}-1\right)\left(\frac{\alpha_{s}^{u z}}{\alpha_{s}^{y z}}-1\right) \\
& \left.+\frac{1}{(\boldsymbol{y}-\boldsymbol{u})^{2}(\boldsymbol{y}-\boldsymbol{z})^{2}}\left(\frac{\alpha_{s}^{y u}}{\alpha_{s}^{x u}}-1\right)\left(\frac{\alpha_{s}^{y z}}{\alpha_{s}^{u z}}-1\right)\right] .
\end{aligned}
$$

By comparing Eq. (59) with Eq. (31), we see that there are extra eight terms resulting from the running coupling corrections, which have a significant impact on the evolution speed of the dipole amplitude. However, these extra terms do not cure the instability issue of the evolution equation. Based on the discussion after Eq. (31), we know that Eq. (59) still has the instabilities caused by double collinear logarithms.

To curve the instability problem, the successive gluon emissions during the rapidity evolution must be ordered in lifetime and longitudinal momentum simultaneously. So, we need to apply the constraints (33) and (34) to the successive gluon emissions, as we have done in the previous section,

$$
\begin{aligned}
\bar{S}_{x y}(\eta)= & S_{x y}^{(0)}+\int \mathrm{d}^{2} z K^{\mathrm{rc}}(\boldsymbol{x}, \boldsymbol{y}, \boldsymbol{z}) \\
& \times \int_{\Theta\left(-\rho_{1}\right)\left|\rho_{1}\right|}^{\eta-\Theta\left(\rho_{1}-\rho\right)\left(\rho_{1}-\rho\right)} \mathrm{d} \eta_{1}\left[\bar{S}_{x z}\left(\eta_{1}\right) \bar{S}_{z y}\left(\eta_{1}\right)-\bar{S}_{x y}\left(\eta_{1}\right)\right] .
\end{aligned}
$$

Note that originally the integral form of the LO BK equation was used in the derivation of the ciBK- $\eta$ equation [31]. In order to achieve the relevant order of accuracy, we use the integral form of the rcBK equation instead of the LO BK equation as the starting point to derive the ciBK- $\eta$ equation. Performing derivatives over $\eta$ in Eq. (61), one can get the exBK- $\eta$ equation

$$
\begin{aligned}
\frac{\partial \bar{S}_{x y}(\eta)}{\partial \eta}= & \int \mathrm{d}^{2} z K^{\mathrm{rc}}(\boldsymbol{x}, \boldsymbol{y}, z) \Theta\left(\eta-\delta_{x y z}\right) \\
& \times\left[\bar{S}_{x z}\left(\eta-\delta_{x z ; r}\right) \bar{S}_{z y}\left(\eta-\delta_{z y ; r}\right)-\bar{S}_{x y}(\eta)\right],
\end{aligned}
$$

which has the same structure as the caBK- $\eta$ equation (49), but with a running-coupling-modified kernel. In terms of the experience from the $Y$ representation, we deduce that the rapidity evolution of the dipole amplitude is also suppressed by the modified kernel, which shall be approved by the numerical calculations in the next section.

\section{Extended full collinearly improved BK equation at $\mathcal{O}\left(\bar{\alpha}_{s}^{2}\right)$}

Based on the discussion in the previous subsection, one can extend the exBK- $\eta$ equation to the full collinearly improved BK equation in $\eta$ by adding the NLO terms from Eq. (59). Before writing down the extended full collinearly improved BK equation in the $\eta$ representation, we need to identify the $\mathcal{O}\left(\bar{\alpha}_{s}^{2}\right)$ piece on the right-hand side of Eq. (62). First, we expand $\bar{S}_{x z}$ and $\bar{S}_{z y}$ to linear order in the rapidity shift, and then use the rcBK equation to replace the derivative terms,

$$
\begin{aligned}
\bar{S}_{x z}\left(\eta-\delta_{x z ; r}\right) \simeq & \bar{S}_{x z}(\eta)-\delta_{x z ; r} \frac{\partial \bar{S}_{x z}(\eta)}{\partial \eta} \\
\simeq & \bar{S}_{x z}(\eta)-\int \mathrm{d}^{2} \boldsymbol{u} K^{\mathrm{rc}}(\boldsymbol{x}, \boldsymbol{z}, \boldsymbol{u}) \delta_{x z ; r} \\
& \times\left[\bar{S}_{x u}(\eta) \bar{S}_{u z}(\eta)-\bar{S}_{x z}(\eta)\right]
\end{aligned}
$$

and

$$
\begin{aligned}
\bar{S}_{z y}\left(\eta-\delta_{z y ; r}\right) \simeq & \bar{S}_{z y}(\eta)-\delta_{z y ; r} \frac{\partial \bar{S}_{z y}(\eta)}{\partial \eta} \\
\simeq & \bar{S}_{z y}(\eta)-\int \mathrm{d}^{2} \boldsymbol{u} K^{\mathrm{rc}}(\boldsymbol{z}, \boldsymbol{y}, \boldsymbol{u}) \\
& \times \delta_{z y ; r}\left[\bar{S}_{z u}(\eta) \bar{S}_{u y}(\eta)-\bar{S}_{z y}(\eta)\right] .
\end{aligned}
$$

Substituting Eqs. (63) and (64) into the nonlinear term in Eq. (62), we get the $\mathcal{O}\left(\bar{\alpha}_{s}^{2}\right)$ piece on the right-hand side of Eq. (62),

$$
\begin{aligned}
& -2 \int \mathrm{d}^{2} z \mathrm{~d}^{2} \boldsymbol{u} K^{\mathrm{rc}}(\boldsymbol{x}, \boldsymbol{y}, \boldsymbol{u}) K^{\mathrm{rc}}(\boldsymbol{u}, \boldsymbol{y}, \boldsymbol{z}) \\
& \quad \times \delta_{u y ; r} \bar{S}_{x u}(\eta)\left[\bar{S}_{u z}(\eta) \bar{S}_{z y}(\eta)-\bar{S}_{u y}(\eta)\right],
\end{aligned}
$$

where we have used the property that the running coupling terms are invariant under $\boldsymbol{x}-\boldsymbol{z} \rightarrow \boldsymbol{z}-\boldsymbol{y}$ (for the detailed derivation, see Appendix B). 
Now we have arrived at the final stage to obtain the extended evolution equation in the $\eta$ representation. Subtracting the $\mathcal{O}\left(\bar{\alpha}_{s}^{2}\right)$ piece from Eq. (62) and adding the $\mathcal{O}\left(\bar{\alpha}_{s}^{2}\right)$ pieces from Eq. (59), we obtain the extended full collinearly improved BK equation in $\eta$ as

$$
\begin{aligned}
\frac{\partial S_{x y}(\eta)}{\partial \eta}= & \frac{\bar{\alpha}_{s}}{2 \pi} \int \mathrm{d}^{2} z \cdot K_{0} \cdot \Theta\left(\eta-\delta_{x y z}\right)\left(\bar{S}_{x z}\left(\eta-\delta_{x z ; r}\right) \bar{S}_{z y}\left(\eta-\delta_{z y ; r}\right)-\bar{S}_{x y}(\eta)\right) \\
& +\frac{\bar{\alpha}_{s}}{2 \pi} \int \mathrm{d}^{2} z \cdot\left(K_{q}+K_{g}\right)\left(\bar{S}_{x z}(\eta) \bar{S}_{z y}(\eta)-\bar{S}_{x y}(\eta)\right) \\
& +\frac{\bar{\alpha}_{s}^{2}}{2 \pi^{2}} \int \mathrm{d}^{2} z \mathrm{~d}^{2} \boldsymbol{u} \cdot K_{3} \cdot \bar{S}_{x u}(\eta)\left(\bar{S}_{u z}(\eta) \bar{S}_{z y}(\eta)-\bar{S}_{u y}(\eta)\right) \\
& +\frac{\bar{\alpha}_{s}^{2}}{8 \pi^{2}} \int \mathrm{d}^{2} \boldsymbol{u} \mathrm{d}^{2} z \cdot K_{1} \cdot\left(\bar{S}_{x u}(\eta) \bar{S}_{u z}(\eta) \bar{S}_{z y}(\eta)-\bar{S}_{x u}(\eta) \bar{S}_{u y}(\eta)\right) \\
& +\frac{\bar{\alpha}_{s}^{2}}{8 \pi^{2}} \frac{N_{f}}{N_{c}} \int \mathrm{d}^{2} \boldsymbol{u} \mathrm{d}^{2} z \cdot K_{f} \cdot\left(\bar{S}_{x z}(\eta) \bar{S}_{u y}(\eta)-\bar{S}_{x u}(\eta) \bar{S}_{u y}(\eta)\right),
\end{aligned}
$$

with

$$
\begin{aligned}
K_{3}= & K_{\mathrm{rc}}+\left[\frac{(\boldsymbol{x}-\boldsymbol{y})^{2}}{(\boldsymbol{x}-\boldsymbol{u})^{2}(\boldsymbol{u}-\boldsymbol{z})^{2}(\boldsymbol{y}-\boldsymbol{z})^{2}}+\frac{(\boldsymbol{x}-\boldsymbol{y})^{2}}{(\boldsymbol{x}-\boldsymbol{u})^{2}(\boldsymbol{y}-\boldsymbol{u})^{2}} \frac{1}{(\boldsymbol{u}-\boldsymbol{z})^{2}}\left(\frac{\alpha_{s}^{u z}}{\alpha_{s}^{y z}}-1\right)\right. \\
& +\frac{(\boldsymbol{x}-\boldsymbol{y})^{2}}{(\boldsymbol{x}-\boldsymbol{u})^{2}(\boldsymbol{y}-\boldsymbol{u})^{2}(\boldsymbol{y}-\boldsymbol{z})^{2}}\left(\frac{\alpha_{s}^{y z}}{\alpha_{s}^{u z}}-1\right)+\frac{(\boldsymbol{u}-\boldsymbol{y})^{2}}{(\boldsymbol{x}-\boldsymbol{u})^{2}(\boldsymbol{u}-\boldsymbol{z})^{2}(\boldsymbol{y}-\boldsymbol{z})^{2}}\left(\frac{\alpha_{s}^{x u}}{\alpha_{s}^{y u}}-1\right) \\
& +\frac{1}{(\boldsymbol{x}-\boldsymbol{u})^{2}(\boldsymbol{u}-\boldsymbol{z})^{2}}\left(\frac{\alpha_{s}^{x u}}{\alpha_{s}^{y u}}-1\right)\left(\frac{\alpha_{s}^{u z}}{\alpha_{s}^{y z}}-1\right)+\frac{1}{(\boldsymbol{x}-\boldsymbol{u})^{2}(\boldsymbol{y}-\boldsymbol{z})^{2}}\left(\frac{\alpha_{s}^{x u}}{\alpha_{s}^{y u}}-1\right)\left(\frac{\alpha_{s}^{y z}}{\alpha_{s}^{u z}}-1\right) \\
& +\frac{1}{(\boldsymbol{u}-\boldsymbol{z})^{2}(\boldsymbol{y}-\boldsymbol{z})^{2}}\left(\frac{\alpha_{s}^{y u}}{\alpha_{s}^{x u}}-1\right)+\frac{1}{(\boldsymbol{y}-\boldsymbol{u})^{2}(\boldsymbol{u}-\boldsymbol{z})^{2}}\left(\frac{\alpha_{s}^{y u}}{\alpha_{s}^{x u}}-1\right)\left(\frac{\alpha_{s}^{u z}}{\alpha_{s}^{y z}}-1\right) \\
& \left.+\frac{1}{(\boldsymbol{y}-\boldsymbol{u})^{2}(\boldsymbol{y}-\boldsymbol{z})^{2}}\left(\frac{\alpha_{s}^{y u}}{\alpha_{s}^{x u}}-1\right)\left(\frac{\alpha_{s}^{y z}}{\alpha_{s}^{u z}}-1\right)\right] \delta_{\boldsymbol{u y} ; r},
\end{aligned}
$$

which is nonlocal in $\eta$ and a stable equation. The double anticollinear logarithmic term in the second line on the right-hand side of Eq. (66) is canceled by the relevant piece generated via the integral over $\boldsymbol{u}$ in the third term. Equation (66) is free of double collinear logarithms, since all of these logarithms are fully included in the first term. By comparing Eq. (66) with Eq. (51), one can find that on the right-hand side of Eq. (66) there are eight extra terms which result from the running coupling corrections in the Taylor expansion of the $S$-matrix. These extra terms play a significant role in the suppression of the evolution speed of the dipole amplitude.

\section{Analytic solution to the exBK- $\eta$ equation in the saturation region}

Let us analytically solve the exBK- $\eta$ equation (62) in the saturation region. In this regime, we know that one of the two daughter dipoles has a similar size as the parent dipole, but the size of the other one is much smaller than that of the parent dipole. Moreover, it is known that the nonlocality is only important for the $S$-matrix which is associated with small size dipole. Therefore, we can reduce Eq. (62) to

$$
\begin{aligned}
\frac{\partial \bar{S}(r, \eta)}{\partial \eta} \simeq & 2 \frac{1}{2 \pi} \bar{S}(r, \eta) \int_{1 / Q_{s}}^{r} \frac{\mathrm{d}^{2} z}{z^{2}} \bar{\alpha}_{s}\left(z^{2}\right) \Theta\left(\eta-\ln \frac{r^{2}}{z^{2}}\right) \\
& \times\left[\bar{S}\left(z, \eta-\ln \frac{r^{2}}{z^{2}}\right)-1\right],
\end{aligned}
$$

where the factor of 2 accounts for the smaller size dipole coming from any one of the two daughter dipoles, and the smallest size of the dipoles $(z)$ is used to as the argument of the QCD coupling.

To solve Eq. (68) analytically in the saturation region, the same strategy is used as that in Sec. III A 3. The one-loop running coupling (10) is used in the calculations. Equation (68) becomes

$$
\frac{\partial \bar{S}(r, \eta)}{\partial \eta} \simeq-\frac{N_{c}}{\pi} \bar{S}(r, \eta) \int_{1 / Q_{s}^{2}}^{r^{2}} \frac{\mathrm{d} z^{2}}{z^{2}} \frac{1}{b \ln \frac{1}{z^{2} \Lambda^{2}}} .
$$


Performing the integral over $z$, we get

$$
\frac{\partial \bar{S}(r, \eta)}{\partial \eta} \simeq-\frac{N_{c}}{b \pi}\left[\ln \left(\ln \frac{Q_{s}^{2}}{\Lambda^{2}}\right)-\ln \left(\ln \frac{1}{r^{2} \Lambda^{2}}\right)\right],
$$

whose solution is

$$
\begin{aligned}
& \bar{S}(r, \eta) \\
& =\exp \left\{-\frac{N_{c}}{b \pi}\left(\eta-\eta_{0}\right)\left[\ln \left(\frac{\sqrt{\lambda^{\prime}}\left(\eta-\eta_{0}\right)+\frac{\sqrt{\bar{\lambda}^{\prime}}}{2} \ln \frac{1}{r^{2} \Lambda^{2}}}{\left(\sqrt{\eta-\eta_{0}}+\frac{\sqrt{\bar{\lambda}^{\prime}}}{2}\right) \ln \frac{1}{r^{2} \Lambda^{2}}}\right)-\frac{1}{2}\right]\right\} \\
& \quad \times \bar{S}\left(r, \eta_{0}\right),
\end{aligned}
$$

with the saturation momentum in the NLO case,

$$
\ln \frac{\bar{Q}_{s}^{2}}{\Lambda^{2}}=\sqrt{\bar{\lambda}^{\prime}\left(\eta-\eta_{0}\right)}+\mathcal{O}\left(\eta^{1 / 6}\right),
$$

and

$$
r^{2} Q_{s}^{2} \simeq\left[r^{2} \bar{Q}_{s}^{2}\right]^{\frac{1}{1+\sqrt{\frac{\bar{\lambda}^{\prime}}{4 \eta}}}}
$$

By comparing Eq. (71) with Eq. (55), one can see that the quadratic rapidity dependence in the exponent of the $S$-matrix is replaced by a linear rapidity dependence once the running coupling corrections are taken into account. This change implies that the evolution speed of the dipole amplitude is suppressed by the NLO corrections.

\section{NUMERICAL ANALYSIS}

To test the analytic results obtained in the previous section, we shall numerically solve the evolution equations in this section. Equations (49) and (62) are integro-differential equations, which can be numerically straightforward solved on a lattice. To simplify the computation, we neglect the impact parameter dependence of the dipole amplitude throughout these numerical calculations, which implies that the dipole amplitude does not depend on the angle, $N(\boldsymbol{r}, Y)=1-S(\boldsymbol{r}, Y)=1-S(|r|, Y)$. Thus, we can view the evolution equations as a set of differential equations and solve them at discrete values of transverse separation. To be more specific, we discretize the dipole transverse size $r$ into 800 points which are equally located in the logarithmic space between $r_{\min }=10^{-8} \mathrm{GeV}^{-1}$ and $r_{\max }=50 \mathrm{GeV}^{-1}$. The GNU Scientific Library (GSL) is a good candidate to solve them, since the GSL contains almost all of the routines required for our purposes, such as the RungeKutta method for solving differential equations, adaptive integral routines for performing numerical integrals, and the cubic spline interpolation codes for interpolating the data points not located on the lattice.

The initial condition for the evolution equations is parametrized at rapidity $\eta=0$. We use the Golec-Biernat and Wusthoff (GBW) parametrization as the initial condition [51],

$$
N^{\mathrm{GBW}}(r, \eta=0)=\left\{1-\exp \left[-\left(\frac{r^{2} Q_{\mathrm{s} 0}^{2}}{4}\right)^{p}\right]\right\}^{1 / p},
$$

with $p=4$, and $Q_{\mathrm{s} 0}=0.362 \mathrm{GeV}[50]$.

For the strong coupling constant, we use the one-loop running coupling (10) with $N_{f}=3$ and $N_{c}=3$. According to the performance of the running coupling in the fit to HERA data [28,30], we choose to use the smallest dipole running coupling prescription, which means that the argument of coupling is the smallest dipole among the parent and daughter dipoles,

$$
\alpha_{s}\left(r_{\min }^{2}\right)=\alpha_{s}\left(\min \left\{(\boldsymbol{x}-\boldsymbol{y})^{2},(\boldsymbol{x}-\boldsymbol{z})^{2},(\boldsymbol{z}-\boldsymbol{y})^{2}\right\}\right) .
$$

We freeze the running coupling $\alpha_{s}\left(r_{\mathrm{fr}}\right)=0.75$ when $r>r_{\mathrm{fr}}$ in order to regularize the infrared behavior.

To show the impact of the running coupling corrections on the speed of the fronts, the saturation exponent is numerically calculated as

$$
\bar{\lambda}=\frac{\mathrm{d} \ln \bar{Q}_{s}^{2}(\eta)}{\mathrm{d} \eta},
$$

where the saturation moment $\bar{Q}_{s}(\eta)$ is determined by $N\left(r=1 / \bar{Q}_{s}, \eta\right)=\kappa$, where $\kappa$ is a constant of order 1 .

The left-hand panel of Fig. 1 gives the solutions of the LO BK and caBK- $\eta$ equations for four different rapidities. By comparing the solutions for each respective rapidity, one can see that the values of the caBK- $\eta$ dipole amplitude are smaller than the LO BK ones, which indicates that the NLO corrections enhanced by the double transverse logarithms suppress the evolution speed of the dipole amplitude. A zoomed-in diagram is provided to clearly show the numerical results in the saturation region. One can see that the evolution is also slowed down in the saturation region. This numerical outcome is consistent with the analytic results in Eqs. (7) and (55), where the analytic solutions of the LO BK and caBK- $\eta$ equations have analogous expressions but different coefficients in the exponent. The coefficient in the caBK- $\eta$ case is smaller than that in the LO BK one, which leads to the caBK- $\eta$ dipole amplitude being smaller than the LO BK one. The right-hand panel of Fig. 1 shows a comparison of the solutions of the LO BK and exBK- $\eta$ equation for four different rapidities. We include a zoomed-in diagram for a clear comparison between the LO BK and exBK- $\eta$ dipole amplitudes in the saturation region. One can see from the zoomed-in diagram that the respective solution of the exBK- $\eta$ equation in each rapidity is much smaller than the LO BK one, which implies that the evolution speed of the dipole amplitude is significantly suppressed. The suppression is much greater than in the caBK- $\eta$ case. This outcome confirms the 

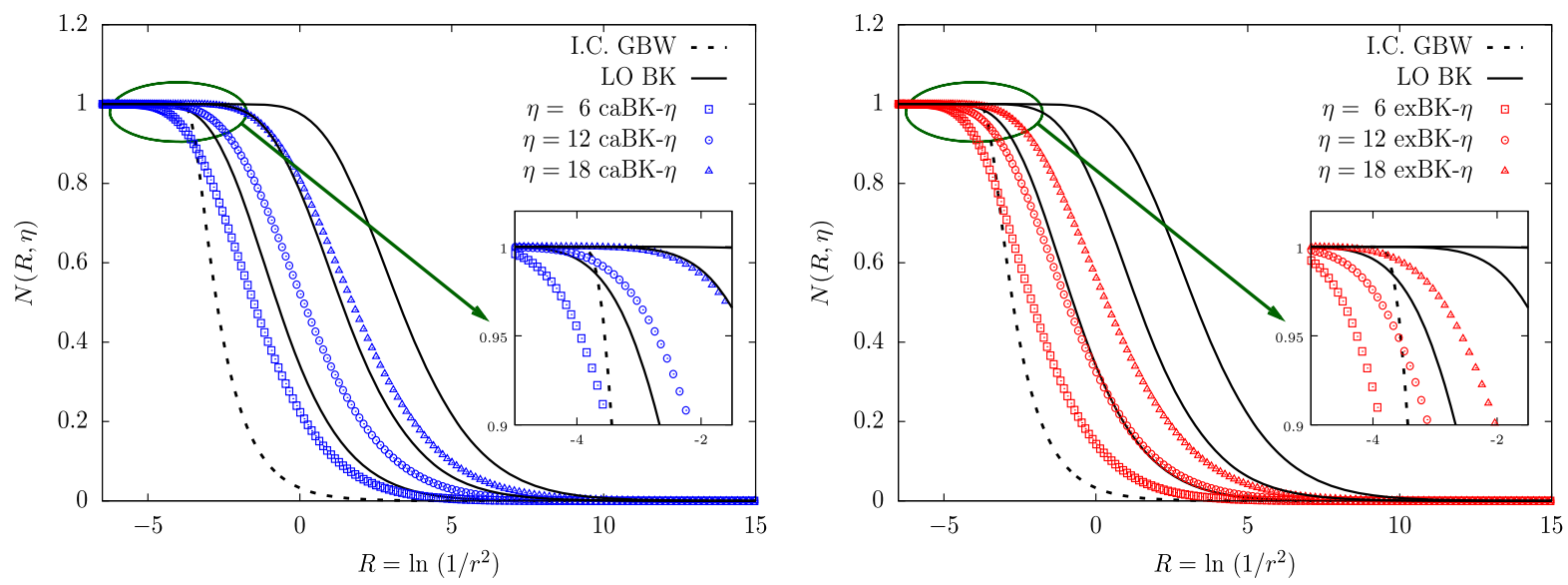

FIG. 1. Numerical solutions to the LO BK, caBK- $\eta$, and exBK- $\eta$ equations for four different rapidities. The left-hand panel shows comparisons of the evolution speed between the LO BK and caBK- $\eta$ dipole amplitudes. The right-hand panel shows comparisons of the evolution speed between the LO BK and exBK- $\eta$ dipole amplitudes. The zoomed-in diagrams show the relevant results in the saturation region.
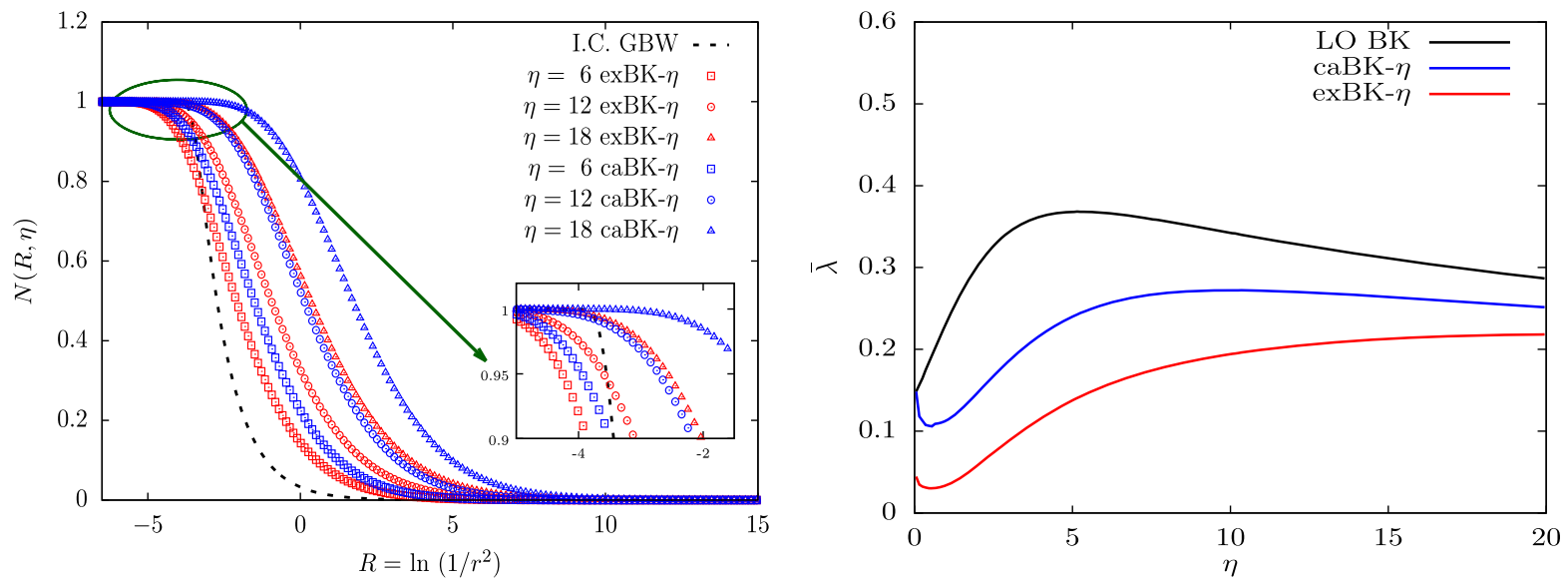

FIG. 2. The left-hand panel gives comparisons of the evolution speed between the caBK- $\eta$ and exBK- $\eta$ dipole amplitudes. The inner diagrams are the zooming in amplitudes in the saturation region. The right-hand panel shows the saturation exponent as a function of $\eta$ in the LO BK, caBK- $\eta$, and exBK- $\eta$ cases.

analytic result in Sec. III B 3, where the dipole amplitude is suppressed by the running coupling corrections.

The left-hand panel of Fig. 2 gives comparisons of the solutions of the caBK- $\eta$ and exBK- $\eta$ equations for four different rapidities. We can see that the respective dipole amplitudes resulting from the exBK- $\eta$ equation are smaller than the ones from the caBK- $\eta$ equation. In particular, it clearly shows the suppression in the saturation region from the inner zoomed-in diagram. This outcome supports the analytic findings in Eq. (71), where the dipole amplitude is further suppressed by the running coupling corrections on top of the double logarithm resummation. Finally, we present the $\eta$ dependence of the saturation exponent predicted by the LO BK, caBK- $\eta$, and exBK- $\eta$ equations in the right-hand panel of Fig. 2. As expected, the $\bar{\lambda}$ resulting from the exBK- $\eta$ equation is the smallest one, which is consistent with the theoretical expectations, since the exBK- $\eta$ equation includes the running coupling contributions on top of the collinear resummations.

\section{ACKNOWLEDGMENTS}

This work is supported by the National Natural Science Foundation of China under Grants No. 11765005, No. 11947119, No. 12061141008, No. 11775097, and No. 11975005; the Fund of Science and Technology Department of Guizhou Province under Grant No. [2019]5653; the Education Department of Guizhou Province under Grant No. KY[2021]131; the National Key Research and Development Program of China under Grants No. 2018YFE0104700 and No. 2018YFE01014800; and the China Postdoctoral Science Foundation under Grant No. 2018M633414. 


\section{APPENDIX A: LOCAL COLLINEARLY IMPROVED BK EQUATION IN $\eta$ WITH RUNNING COUPLING CORRECTIONS IN THE TAYLOR EXPANSION}

We give a detailed derivation of the collinearly improved BK equation in the $\eta$ representation by using the rcBK equation to expand out $S_{x z}(Y)$ in Eq. (56) and $S_{z y}(Y)$ in Eq. (57). To simplify the calculations, we rewrite the running coupling evolution kernel (58) as

$$
K^{\mathrm{rc}}(\boldsymbol{x}, \boldsymbol{y}, \boldsymbol{z})=\frac{\bar{\alpha}_{s}}{2 \pi}\left(K_{0}+K_{q^{\prime}}\right),
$$

with

$$
K_{q^{\prime}}=\frac{1}{(\boldsymbol{x}-z)^{2}}\left(\frac{\alpha_{s}^{x z}}{\alpha_{s}^{y z}}-1\right)+\frac{1}{(\boldsymbol{y}-z)^{2}}\left(\frac{\alpha_{s}^{y z}}{\alpha_{s}^{x z}}-1\right)
$$

The full NLO BK equation (15) is our starting point for this derivation. Using Eq. (A1), Eq. (15) is rewritten as

$$
\begin{aligned}
\frac{\partial S_{x y}(Y)}{\partial Y}= & \int \mathrm{d}^{2} z K^{\mathrm{rc}}(\boldsymbol{x}, \boldsymbol{y}, \boldsymbol{z})\left[S_{x z}(Y) S_{z y}(Y)-S_{x y}(Y)\right]+\frac{\bar{\alpha}_{s}}{2 \pi} \int \mathrm{d}^{2} z \cdot K_{g} \cdot\left[S_{x z}(Y) S_{z y}(Y)-S_{x y}(Y)\right] \\
& +\frac{\bar{\alpha}_{s}^{2}}{8 \pi^{2}} \int \mathrm{d}^{2} \boldsymbol{u} \mathrm{d}^{2} z \cdot K_{1} \cdot\left[S_{x u}(Y) S_{u z}(Y) S_{z y}(Y)-S_{x u}(Y) S_{u y}(Y)\right] \\
& +\frac{\bar{\alpha}_{s}^{2}}{8 \pi^{2}} \frac{N_{f}}{N_{c}} \int \mathrm{d}^{2} \boldsymbol{u} \mathrm{d}^{2} z \cdot K_{f} \cdot\left[S_{x z}(Y) S_{u y}(Y)-S_{x u}(Y) S_{u y}(Y)\right] .
\end{aligned}
$$

Note that the kernel $K^{\text {rc }}$ is of the order of $\mathcal{O}\left(\bar{\alpha}_{s}\right)$, which means that the expansions of $S_{x z}(Y)$ and $S_{z y}(Y)$ in Eqs. (56) and (57) are equivalent to adding a term of order $\mathcal{O}\left(\bar{\alpha}_{s}\right)$. Thus, we can deduce the rapidity shift by using a similar scheme in the LO expansion case. For the first term (denoted as $T_{\text {rc }}$ ) on the right-hand side of Eq. (A3), we have

$$
\begin{aligned}
T_{\mathrm{rc}} & =\int \mathrm{d}^{2} z K^{\mathrm{rc}}(\boldsymbol{x}, \boldsymbol{y}, \boldsymbol{z})\left[S_{x z}(Y) S_{z y}(Y)-S_{x y}(Y)\right] \\
& =\int \mathrm{d}^{2} z K^{\mathrm{rc}}(\boldsymbol{x}, \boldsymbol{y}, \boldsymbol{z})\left[\bar{S}_{\boldsymbol{x} z}\left(\eta+\ln \frac{(\boldsymbol{x}-\boldsymbol{z})^{2}}{(\boldsymbol{x}-\boldsymbol{y})^{2}}\right) \bar{S}_{z y}\left(\eta+\ln \frac{(\boldsymbol{y}-\boldsymbol{z})^{2}}{(\boldsymbol{x}-\boldsymbol{y})^{2}}\right)-\bar{S}_{x \boldsymbol{y}}(\eta)\right] .
\end{aligned}
$$

Substituting Eqs. (56) and (57) into Eq. (A4), one can get

$$
\begin{aligned}
T_{\mathrm{rc}}= & \int \mathrm{d}^{2} z K^{\mathrm{rc}}(\boldsymbol{x}, \boldsymbol{y}, \boldsymbol{z})\left\{\left[\bar{S}_{x z}(\eta)+\int \mathrm{d}^{2} \boldsymbol{u} K^{\mathrm{rc}}(\boldsymbol{x}, \boldsymbol{z}, \boldsymbol{u}) \ln \frac{(\boldsymbol{x}-\boldsymbol{z})^{2}}{(\boldsymbol{x}-\boldsymbol{y})^{2}}\left[\bar{S}_{\boldsymbol{x} \boldsymbol{u}}(\eta) \bar{S}_{\boldsymbol{u z}}(\eta)-\bar{S}_{\boldsymbol{x} z}(\eta)\right]\right]\right. \\
& \left.\times\left[\bar{S}_{z y}(\eta)+\int \mathrm{d}^{2} \boldsymbol{u} K^{\mathrm{rc}}(\boldsymbol{z}, \boldsymbol{y}, \boldsymbol{u}) \ln \frac{(\boldsymbol{z}-\boldsymbol{y})^{2}}{(\boldsymbol{x}-\boldsymbol{y})^{2}}\left[\bar{S}_{z \boldsymbol{u}}(\eta) \bar{S}_{u \boldsymbol{y}}(\eta)-\bar{S}_{z y}(\eta)\right]\right]-\bar{S}_{x \boldsymbol{y}}(\eta)\right\} .
\end{aligned}
$$

After some algebraic calculations, Eq. (A5) becomes

$$
\begin{aligned}
T_{\mathrm{rc}}= & \int \mathrm{d}^{2} z K^{\mathrm{rc}}(\boldsymbol{x}, \boldsymbol{y}, \boldsymbol{z})\left\{\left[\bar{S}_{x z}(\eta) \bar{S}_{z \boldsymbol{y}}(\eta)-\bar{S}_{x \boldsymbol{y}}(\eta)\right]+\bar{S}_{\boldsymbol{x} z}(\eta) \int \mathrm{d}^{2} \boldsymbol{u} K^{\mathrm{rc}}(\boldsymbol{z}, \boldsymbol{y}, \boldsymbol{u}) \ln \frac{(\boldsymbol{z}-\boldsymbol{y})^{2}}{(\boldsymbol{x}-\boldsymbol{y})^{2}}\left[\bar{S}_{z \boldsymbol{u}}(\eta) \bar{S}_{u y}(\eta)-\bar{S}_{z \boldsymbol{y}}(\eta)\right]\right. \\
& +\bar{S}_{z \boldsymbol{y}}(\eta) \int \mathrm{d}^{2} \boldsymbol{u} K^{\mathrm{rc}}(\boldsymbol{x}, \boldsymbol{z}, \boldsymbol{u}) \ln \frac{(\boldsymbol{x}-\boldsymbol{z})^{2}}{(\boldsymbol{x}-\boldsymbol{y})^{2}}\left[\bar{S}_{x \boldsymbol{u}}(\eta) \bar{S}_{u z}(\eta)-\bar{S}_{x z}(\eta)\right] \\
& \left.+\int \mathrm{d}^{2} \boldsymbol{u} \mathrm{d}^{2} \boldsymbol{v} K^{\mathrm{rc}}(\boldsymbol{x}, \boldsymbol{z}, \boldsymbol{u}) K^{\mathrm{rc}}(\boldsymbol{z}, \boldsymbol{y}, \boldsymbol{v}) \ln \frac{(\boldsymbol{x}-\boldsymbol{z})^{2}}{(\boldsymbol{x}-\boldsymbol{y})^{2}} \ln \frac{(\boldsymbol{z}-\boldsymbol{y})^{2}}{(\boldsymbol{x}-\boldsymbol{y})^{2}}\left[\bar{S}_{x \boldsymbol{u}}(\eta) \bar{S}_{u z}(\eta)-\bar{S}_{x z}(\eta)\right]\left[\bar{S}_{z \boldsymbol{v}}(\eta) \bar{S}_{\boldsymbol{y} \boldsymbol{y}}(\eta)-\bar{S}_{z \boldsymbol{y}}(\eta)\right]\right\} .
\end{aligned}
$$

Using the property that the running coupling terms are invariant under $\boldsymbol{x}-\boldsymbol{z} \rightarrow \boldsymbol{z}-\boldsymbol{y}$, we can see that the second term is equal to the third term in the braces of Eq. (A6). In addition, the fourth term in the braces of Eq. (A6) is of the order of $\mathcal{O}\left(\bar{\alpha}_{s}^{2}\right)$. It becomes $\mathcal{O}\left(\bar{\alpha}_{s}^{3}\right)$ because of an extra $K^{\text {rc }}$ factor in front the braces and can be discarded. Therefore, Eq. (A6) can be reduced to 


$$
\begin{aligned}
T_{\mathrm{rc}}= & \int \mathrm{d}^{2} z K^{\mathrm{rc}}(\boldsymbol{x}, \boldsymbol{y}, \boldsymbol{z})\left[\bar{S}_{\boldsymbol{x} z}(\eta) \bar{S}_{z \boldsymbol{y}}(\eta)-\bar{S}_{\boldsymbol{x} \boldsymbol{y}}(\eta)\right] \\
& +2 \int \mathrm{d}^{2} z \mathrm{~d}^{2} \boldsymbol{u} K^{\mathrm{rc}}(\boldsymbol{x}, \boldsymbol{y}, \boldsymbol{z}) K^{\mathrm{rc}}(\boldsymbol{z}, \boldsymbol{y}, \boldsymbol{u}) \ln \frac{(\boldsymbol{z}-\boldsymbol{y})^{2}}{(\boldsymbol{x}-\boldsymbol{y})^{2}} \bar{S}_{x z}(\eta)\left[\bar{S}_{z \boldsymbol{u}}(\eta) \bar{S}_{u y}(\eta)-\bar{S}_{z y}(\eta)\right] .
\end{aligned}
$$

In order to keep consistency with the physics picture, we relabel the integral variables in the second term on the right-hand side of Eq. (A7) through the variable transformation $\boldsymbol{u} \leftrightarrow z$. Then, Eq. (A7) becomes

$$
\begin{aligned}
T_{\mathrm{rc}}= & \int \mathrm{d}^{2} z K^{\mathrm{rc}}(\boldsymbol{x}, \boldsymbol{y}, \boldsymbol{z})\left[\bar{S}_{\boldsymbol{x} z}(\eta) \bar{S}_{z \boldsymbol{y}}(\eta)-\bar{S}_{x \boldsymbol{y}}(\eta)\right] \\
& +2 \int \mathrm{d}^{2} z \mathrm{~d}^{2} \boldsymbol{u} K^{\mathrm{rc}}(\boldsymbol{x}, \boldsymbol{y}, \boldsymbol{u}) K^{\mathrm{rc}}(\boldsymbol{u}, \boldsymbol{y}, \boldsymbol{z}) \ln \frac{(\boldsymbol{u}-\boldsymbol{y})^{2}}{(\boldsymbol{x}-\boldsymbol{y})^{2}} \bar{S}_{x \boldsymbol{u}}(\eta)\left[\bar{S}_{\boldsymbol{u} z}(\eta) \bar{S}_{z y}(\eta)-\bar{S}_{\boldsymbol{u y}}(\eta)\right] .
\end{aligned}
$$

The last three terms on the right-hand side of Eq. (A3) are of order of $\mathcal{O}\left(\bar{\alpha}_{s}^{2}\right)$. For these three terms, we can simply replace the rapidity shift as $S_{x u}(Y) \rightarrow S_{x u}(\eta)$ since the rapidity shift makes them of order $\mathcal{O}\left(\bar{\alpha}_{s}^{3}\right)$, which can be safely neglected in our study. Combining these terms with $T_{\text {rc }}$ in Eq. (A8), one can obtain

$$
\begin{aligned}
\frac{\partial \bar{S}_{x y}(\eta)}{\partial \eta}= & \int \mathrm{d}^{2} z K^{\mathrm{rc}}(\boldsymbol{x}, \boldsymbol{y}, \boldsymbol{z})\left[\bar{S}_{x z}(\eta) \bar{S}_{z y}(\eta)-\bar{S}_{x y}(\eta)\right] \\
& +2 \int \mathrm{d}^{2} z \mathrm{~d}^{2} \boldsymbol{u} K^{\mathrm{rc}}(\boldsymbol{x}, \boldsymbol{y}, \boldsymbol{u}) K^{\mathrm{rc}}(\boldsymbol{u}, \boldsymbol{y}, \boldsymbol{z}) \ln \frac{(\boldsymbol{u}-\boldsymbol{y})^{2}}{(\boldsymbol{x}-\boldsymbol{y})^{2}} \bar{S}_{x u}(\eta)\left[\bar{S}_{u z}(\eta) \bar{S}_{z y}(\eta)-\bar{S}_{u y}(\eta)\right] \\
& +\frac{\bar{\alpha}_{s}}{2 \pi} \int \mathrm{d}^{2} z \cdot K_{g} \cdot\left[\bar{S}_{x z}(\eta) \bar{S}_{z y}(\eta)-\bar{S}_{x y}(\eta)\right] \\
& +\frac{\bar{\alpha}_{s}^{2}}{8 \pi^{2}} \int \mathrm{d}^{2} \boldsymbol{u} \mathrm{d}^{2} z \cdot K_{1} \cdot\left[\bar{S}_{x u}(\eta) \bar{S}_{u z}(\eta) \bar{S}_{z y}(\eta)-\bar{S}_{x u}(\eta) \bar{S}_{u y}(\eta)\right] \\
& +\frac{\bar{\alpha}_{s}^{2}}{8 \pi^{2}} \frac{N_{f}}{N_{c}} \int \mathrm{d}^{2} \boldsymbol{u} \mathrm{d}^{2} z \cdot K_{f} \cdot\left[\bar{S}_{x z}(\eta) \bar{S}_{u y}(\eta)-\bar{S}_{x u}(\eta) \bar{S}_{u y}(\eta)\right]
\end{aligned}
$$

The first integral term (denoted as $T_{\mathrm{LK}}$ ) and second integral term (denoted as $T_{\mathrm{SK}}$ ) in Eq. (A9) contain a linear $K^{\mathrm{rc}}$ factor and quadratic $K^{\mathrm{rc}}$ factor, respectively. For a clear comparison with the LO expansion case, we expand out these two terms by using Eq. (A1),

$$
\begin{aligned}
T_{\mathrm{LK}} & =\int \mathrm{d}^{2} z K^{\mathrm{rc}}(\boldsymbol{x}, \boldsymbol{y}, \boldsymbol{z})\left[\bar{S}_{x z}(\eta) \bar{S}_{z y}(\eta)-\bar{S}_{x y}(\eta)\right] \\
& =\frac{\bar{\alpha}_{s}}{2 \pi} \int \mathrm{d}^{2} z \cdot K_{0} \cdot\left[\bar{S}_{x z}(\eta) \bar{S}_{z y}(\eta)-\bar{S}_{x y}(\eta)\right]+\frac{\bar{\alpha}_{s}}{2 \pi} \int \mathrm{d}^{2} z \cdot K_{q} \cdot\left[\bar{S}_{x z}(\eta) \bar{S}_{z y}(\eta)-\bar{S}_{x y}(\eta)\right],
\end{aligned}
$$

and

$$
\begin{aligned}
T_{\mathrm{SK}}= & 2 \int \mathrm{d}^{2} z \mathrm{~d}^{2} \boldsymbol{u} K^{\mathrm{rc}}(\boldsymbol{x}, \boldsymbol{y}, \boldsymbol{u}) K^{\mathrm{rc}}(\boldsymbol{u}, \boldsymbol{y}, \boldsymbol{z}) \ln \frac{(\boldsymbol{u}-\boldsymbol{y})^{2}}{(\boldsymbol{x}-\boldsymbol{y})^{2}} \bar{S}_{x \boldsymbol{u}}(\eta)\left[\bar{S}_{\boldsymbol{u z}}(\eta) \bar{S}_{z \boldsymbol{y}}(\eta)-\bar{S}_{\boldsymbol{u y}}(\eta)\right] \\
= & 2 \int \mathrm{d}^{2} z \mathrm{~d}^{2} \boldsymbol{u} \frac{\bar{\alpha}_{s}}{2 \pi}\left[\frac{(\boldsymbol{x}-\boldsymbol{y})^{2}}{(\boldsymbol{x}-\boldsymbol{u})^{2}(\boldsymbol{y}-\boldsymbol{u})^{2}}+\frac{1}{(\boldsymbol{x}-\boldsymbol{u})^{2}}\left(\frac{\alpha_{s}^{x u}}{\alpha_{s}^{y u}}-1\right)+\frac{1}{(\boldsymbol{y}-\boldsymbol{u})^{2}}\left(\frac{\alpha_{s}^{y u}}{\alpha_{s}^{x u}}-1\right)\right] \\
& \times \frac{\bar{\alpha}_{s}}{2 \pi}\left[\frac{(\boldsymbol{u}-\boldsymbol{y})^{2}}{(\boldsymbol{u}-\boldsymbol{z})^{2}(\boldsymbol{y}-\boldsymbol{z})^{2}}+\frac{1}{(\boldsymbol{u}-\boldsymbol{z})^{2}}\left(\frac{\alpha_{s}^{u z}}{\alpha_{s}^{y z}}-1\right)+\frac{1}{(\boldsymbol{y}-\boldsymbol{z})^{2}}\left(\frac{\alpha_{s}^{y z}}{\alpha_{s}^{u z}}-1\right)\right] \\
& \times \ln \frac{(\boldsymbol{u}-\boldsymbol{y})^{2}}{(\boldsymbol{x}-\boldsymbol{y})^{2}} \bar{S}_{\boldsymbol{x} \boldsymbol{u}}(\eta)\left[\bar{S}_{\boldsymbol{u} z}(\eta) \bar{S}_{z \boldsymbol{y}}(\eta)-\bar{S}_{u \boldsymbol{y}}(\eta)\right] .
\end{aligned}
$$

After some algebraic calculations, Eq. (A11) becomes 


$$
T_{\mathrm{SK}}=\frac{\bar{\alpha}_{s}^{2}}{2 \pi^{2}} \int \mathrm{d}^{2} z \mathrm{~d}^{2} \boldsymbol{u} \cdot K_{\mathrm{rc}} \cdot \bar{S}_{x u}(\eta)\left[\bar{S}_{u z}(\eta) \bar{S}_{z y}(\eta)-\bar{S}_{u y}(\eta)\right]
$$

where $K_{\mathrm{rc}}$ is defined in Eq. (60).

Substituting Eqs. (A10) and (A12) into Eq. (A9), one can obtain a semifinished collinearly improved BK equation in $\eta$,

$$
\begin{aligned}
\frac{\partial \bar{S}_{x y}(\eta)}{\partial \eta}= & \frac{\bar{\alpha}_{s}}{2 \pi} \int \mathrm{d}^{2} z \cdot K_{0} \cdot\left[\bar{S}_{x z}(\eta) \bar{S}_{z y}(\eta)-\bar{S}_{x y}(\eta)\right]+\frac{\bar{\alpha}_{s}}{2 \pi} \int \mathrm{d}^{2} z \cdot\left(K_{q}+K_{g}\right) \cdot\left[\bar{S}_{x z}(\eta) \bar{S}_{z y}(\eta)-\bar{S}_{x y}(\eta)\right] \\
& +\frac{\bar{\alpha}_{s}^{2}}{2 \pi^{2}} \int \mathrm{d}^{2} z \mathrm{~d}^{2} \boldsymbol{u} \cdot K_{\mathrm{rc}} \cdot \bar{S}_{x u}(\eta)\left[\bar{S}_{u z}(\eta) \bar{S}_{z y}(\eta)-\bar{S}_{u y}(\eta)\right]+\frac{\bar{\alpha}_{s}^{2}}{8 \pi^{2}} \int \mathrm{d}^{2} \boldsymbol{u} \mathrm{d}^{2} z \cdot K_{1} \cdot\left[\bar{S}_{x u}(\eta) \bar{S}_{u z}(\eta) \bar{S}_{z y}(\eta)-\bar{S}_{x u}(\eta) \bar{S}_{u y}(\eta)\right] \\
& +\frac{\bar{\alpha}_{s}^{2}}{8 \pi^{2}} \frac{N_{f}}{N_{c}} \int \mathrm{d}^{2} \boldsymbol{u} \mathrm{d}^{2} z \cdot K_{f} \cdot\left[\bar{S}_{x z}(\eta) \bar{S}_{u y}(\eta)-\bar{S}_{x u}(\eta) \bar{S}_{u y}(\eta)\right]
\end{aligned}
$$

\section{APPENDIX B: IDENTIFYING AN $\mathcal{O}\left(\bar{\alpha}_{s}^{2}\right)$ PIECE IN EQ. (62)}

In this appendix, we give the details of the derivation to identify an $\mathcal{O}\left(\bar{\alpha}_{s}^{2}\right)$ piece on the right-hand side of Eq. (62). Substituting Eqs. (63) and (64) into Eq. (62) and neglecting the step function in it, one can get

$$
\begin{aligned}
\frac{\partial \bar{S}_{x y}(\eta)}{\partial \eta}= & \int \mathrm{d}^{2} z K^{\mathrm{rc}}(\boldsymbol{x}, \boldsymbol{y}, \boldsymbol{z})\left\{\left[\bar{S}_{x z}(\eta)-\int \mathrm{d}^{2} \boldsymbol{u} K^{\mathrm{rc}}(\boldsymbol{x}, \boldsymbol{z}, \boldsymbol{u}) \delta_{x z ; r}\left[\bar{S}_{x u}(\eta) \bar{S}_{u z}(\eta)-\bar{S}_{x z}(\eta)\right]\right]\right. \\
& \times\left[\bar{S}_{z y}(\eta)-\int \mathrm{d}^{2} \boldsymbol{u} K^{\mathrm{rc}}(\boldsymbol{z}, \boldsymbol{y}, \boldsymbol{u}) \delta_{z y ; r}\left[\bar{S}_{z u}(\eta) \bar{S}_{\boldsymbol{u} \boldsymbol{y}}(\eta)-\bar{S}_{z y}(\eta)\right]-\bar{S}_{x \boldsymbol{y}}(\eta)\right\} .
\end{aligned}
$$

Expanding the terms in the braces on the right-hand side of Eq. (B1), we obtain

$$
\begin{aligned}
\frac{\partial \bar{S}_{x y}(\eta)}{\partial \eta}= & \int \mathrm{d}^{2} z K^{\mathrm{rc}}(\boldsymbol{x}, \boldsymbol{y}, \boldsymbol{z})\left\{\left[\bar{S}_{x z}(\eta) \bar{S}_{z \boldsymbol{y}}(\eta)-\bar{S}_{x y}(\eta)\right]-\bar{S}_{x z}(\eta) \int \mathrm{d}^{2} \boldsymbol{u} K^{\mathrm{rc}}(\boldsymbol{z}, \boldsymbol{y}, \boldsymbol{u}) \delta_{z y ; r}\right. \\
& \times\left[\bar{S}_{z u}(\eta) \bar{S}_{u \boldsymbol{y}}(\eta)-\bar{S}_{z y}(\eta)\right]-\bar{S}_{z y}(\eta) \int \mathrm{d}^{2} \boldsymbol{u} K^{\mathrm{rc}}(\boldsymbol{x}, \boldsymbol{z}, \boldsymbol{u}) \delta_{x z ; r}\left[\bar{S}_{x \boldsymbol{u}}(\eta) \bar{S}_{u z}(\eta)-\bar{S}_{x z}(\eta)\right] \\
& \left.+\int \mathrm{d}^{2} \boldsymbol{u} \mathrm{d}^{2} \boldsymbol{v} K^{\mathrm{rc}}(\boldsymbol{x}, \boldsymbol{z}, \boldsymbol{u}) K^{\mathrm{rc}}(\boldsymbol{z}, \boldsymbol{y}, \boldsymbol{v}) \delta_{x z ; r} \delta_{z y ; r}\left[\bar{S}_{x \boldsymbol{u}}(\eta) \bar{S}_{\boldsymbol{u} z}(\eta)-\bar{S}_{x z}(\eta)\right]\left[\bar{S}_{z \boldsymbol{v}}(\eta) \bar{S}_{v \boldsymbol{y}}(\eta)-\bar{S}_{z y}(\eta)\right]\right\},
\end{aligned}
$$

which has a similar structure as Eq. (A6), so we use the same scheme to simplify it. Using the property that the running coupling terms are invariant under $\boldsymbol{x}-\boldsymbol{z} \rightarrow \boldsymbol{z}-\boldsymbol{y}$ and discarding the last term of the order of $\mathcal{O}\left(\bar{\alpha}_{s}^{3}\right)$, Eq. (B2) can be reduced to

$$
\begin{aligned}
\frac{\partial \bar{S}_{x \boldsymbol{y}}(\eta)}{\partial \eta}= & \int \mathrm{d}^{2} z K^{\mathrm{rc}}(\boldsymbol{x}, \boldsymbol{y}, \boldsymbol{z})\left[\bar{S}_{x z}(\eta) \bar{S}_{z \boldsymbol{y}}(\eta)-\bar{S}_{x y}(\eta)\right] \\
& -2 \int \mathrm{d}^{2} \boldsymbol{z} \mathrm{d}^{2} \boldsymbol{u} K^{\mathrm{rc}}(\boldsymbol{x}, \boldsymbol{y}, \boldsymbol{z}) K^{\mathrm{rc}}(\boldsymbol{z}, \boldsymbol{y}, \boldsymbol{u}) \delta_{z y ; r} \bar{S}_{x z}(\eta)\left[\bar{S}_{z \boldsymbol{u}}(\eta) \bar{S}_{u y}(\eta)-\bar{S}_{z y}(\eta)\right]
\end{aligned}
$$

In order to keep consistency with the physics picture mentioned in the previous sections, we relabel the integral variables according to $\boldsymbol{u} \leftrightarrow \boldsymbol{z}$ for the second term on the right-hand side Eq. (A12), which becomes

$$
\begin{aligned}
\frac{\partial \bar{S}_{x y}(\eta)}{\partial \eta}= & \int \mathrm{d}^{2} z K^{\mathrm{rc}}(\boldsymbol{x}, \boldsymbol{y}, \boldsymbol{z})\left[\bar{S}_{x z}(\eta) \bar{S}_{z y}(\eta)-\bar{S}_{x \boldsymbol{y}}(\eta)\right] \\
& -2 \int \mathrm{d}^{2} \boldsymbol{z} \mathrm{d}^{2} \boldsymbol{u} K^{\mathrm{rc}}(\boldsymbol{x}, \boldsymbol{y}, \boldsymbol{u}) K^{\mathrm{rc}}(\boldsymbol{u}, \boldsymbol{y}, \boldsymbol{z}) \delta_{\boldsymbol{u} ; r} \bar{S}_{x \boldsymbol{u}}(\eta)\left[\bar{S}_{\boldsymbol{u} z}(\eta) \bar{S}_{z \boldsymbol{y}}(\eta)-\bar{S}_{\boldsymbol{u y}}(\eta)\right]
\end{aligned}
$$

It is clear that the $\mathcal{O}\left(\bar{\alpha}_{s}^{2}\right)$ piece in the above equation is

$$
-2 \int \mathrm{d}^{2} z \mathrm{~d}^{2} \boldsymbol{u} K^{\mathrm{rc}}(\boldsymbol{x}, \boldsymbol{y}, \boldsymbol{u}) K^{\mathrm{rc}}(\boldsymbol{u}, \boldsymbol{y}, \boldsymbol{z}) \delta_{\boldsymbol{u} y ; r} \bar{S}_{\boldsymbol{x} \boldsymbol{u}}(\eta)\left[\bar{S}_{\boldsymbol{u z}}(\eta) \bar{S}_{z \boldsymbol{y}}(\eta)-\bar{S}_{\boldsymbol{u y}}(\eta)\right]
$$


[1] I. Balitsky, Nucl. Phys. B463, 99 (1996).

[2] J. Jalilian-Marian, A. Kovner, A. Leonidov, and H. Weigert, Nucl. Phys. B504, 415 (1997).

[3] J. Jalilian-Marian, A. Kovner, A. Leonidov, and H. Weigert, Phys. Rev. D 59, 014014 (1998).

[4] E. Iancu, A. Leonidov, and L. McLerran, Nucl. Phys. A692, 583 (2001).

[5] E. Ferreiro, E. Iancu, A. Leonidov, and L. McLerran, Nucl. Phys. A703, 489 (2002).

[6] Y. Kovchegov, Phys. Rev. D 60, 034008 (1999); 61, 074018 (2000).

[7] E. Iancu, K. Itakura, and S. Munier, Phys. Lett. B 590, 199 (2004).

[8] M. Kozlov, A. Shoshi, and W. Xiang, J. High Energy Phys. 10 (2007) 020.

[9] D. Kkarzeev and E. Levin, Phys. Lett. B 523, 79 (2001).

[10] A. Dumitru, A. Hayashigaki, and J. Jalilian-Marian, Nucl. Phys. A765, 464 (2006).

[11] E. Levin and A. Rezaeian, Phys. Rev. D 82, 074016 (2010).

[12] G. Chirilli, B. Xiao, and F. Yuan, Phys. Rev. Lett. 108, 122301 (2012).

[13] T. Lappi and H. Mäntysaari, Phys. Rev. D 88, 114020 (2013).

[14] J. Albacete, N. Armesto, J. Milhano, and C. Salgado, Phys. Rev. D 80, 034031 (2009).

[15] J. Albacete, N. Armesto, J. Milhano, P. Quiroga-Arias, and C. Salgado, Eur. Phys. J. C 71, 1705 (2011).

[16] B. Ducloue, H. Hanninen, T. Lappi, and Y. Zhu, Phys. Rev. D 96, 094017 (2017).

[17] I. Balitsky, Phys. Rev. D 75, 014001 (2007).

[18] Y. Kovchegov and H. Weigert, Nucl. Phys. A784, 188 (2007).

[19] I. Balitsky and G. Chirilli, Phys. Rev. D 77, 014019 (2008).

[20] A. Kovner, M. Lublinsky, and Y. Mulian, Phys. Rev. D 89, 061704 (2014).

[21] A. Kovner, M. Lublinsky, and Y. Mulian, J. High Energy Phys. 04 (2014) 030.

[22] A. Kovner, M. Lublinsky, and Y. Mulian, J. High Energy Phys. 08 (2014) 114.

[23] D. Zheng and J. Zhou, J. High Energy Phys. 11 (2019) 177.

[24] T. Lappi and H. Mäntysaari, Phys. Rev. D 91, 074016 (2015).
[25] T. Lappi and H. Mäntysaari, Phys. Rev. D 93, 094004 (2016).

[26] G. Beuf, Phys. Rev. D 89, 074039 (2014).

[27] E. Iancu, J. Madrigal, A. Mueller, G. Soyez, and D. Triantafyllopoulos, Phys. Lett. B 744, 293 (2015).

[28] E. Iancu, J. Madrigal, A. Mueller, G. Soyez, and D. Triantafyllopoulos, Phys. Lett. B 750, 643 (2015).

[29] Y. Cai, W. Xiang, M. Wang, and D. Zhou, Chin. Phys. C 44, 074110 (2020).

[30] W. Xiang, M. Wang, Y. Cai, and D. Zhou, Chin. Phys. C 45, 014103 (2021).

[31] B. Ducloue, E. Iancu, A. Mueller, G. Soyez, and D. Triantafyllopoulos, J. High Energy Phys. 04 (2019) 081.

[32] V. Fadin, M. Kotsky, and R. Fiore, Phys. Lett. B 359, 181 (1995).

[33] G. Camici and M. Ciafaloni, Phys. Lett. B 412, 396 (1997).

[34] M. Ciafaloni and G. Camici, Phys. Lett. B 430, 349 (1998).

[35] I. Balitsky and G. Chirilli, Phys. Rev. D 83, 031502 (2011).

[36] I. Balitsky and G. Chirilli, Phys. Rev. D 87, 014013 (2013).

[37] G. Beuf, Phys. Rev. D 85, 034039 (2012).

[38] G. Beuf, Phys. Rev. D 94, 054016 (2016).

[39] G. Beuf, Phys. Rev. D 96, 074033 (2017).

[40] G. Beuf, H. Hanninen, and T. Lappi, Phys. Rev. D 102, 074028 (2020).

[41] A. Mueller, arXiv:hep-ph/0111244.

[42] W. Xiang, Phys. Rev. D 79, 014012 (2009).

[43] E. Levin and K. Tuchin, Nucl. Phys. B573, 833 (2000).

[44] W. Xiang, S. Cai, and D. Zhou, Phys. Rev. D 95, 116009 (2017).

[45] C. Contreras, E. Levin, R. Meneses, and I. Potashnikova, Phys. Rev. D 94, 114028 (2016).

[46] W. Xiang, Y. Cai, M. Wang, and D. Zhou, Phys. Rev. D 101, 076005 (2020).

[47] J. Albacete, Nucl. Phys. A957, 71 (2017).

[48] J. Cepila, J. Contreras, and M. Matas, Phys. Rev. D 100, 054015 (2019).

[49] J. Albacete and Yu. V. Kovchegov, Phys. Rev. D 75, 125021 (2007).

[50] B. Ducloue, E. Iancu, G. Soyez, and D. Triantafyllopoulos, Phys. Lett. B 803, 135305 (2020).

[51] K. Golec-Biernat and M. Wusthoff, Phys. Rev. D 59, 014017 (1998). 